\title{
Serotonergic Modulation of Inhibitory Input to Lateral Horn Affects Odor-Mediated Attraction in D. melanogaster
}

Ayad Auda

Follow this and additional works at: https://researchrepository.wvu.edu/etd

\section{Recommended Citation}

Auda, Ayad, "Serotonergic Modulation of Inhibitory Input to Lateral Horn Affects Odor-Mediated Attraction in D. melanogaster" (2017). Graduate Theses, Dissertations, and Problem Reports. 5131.

https://researchrepository.wvu.edu/etd/5131

This Thesis is protected by copyright and/or related rights. It has been brought to you by the The Research Repository @ WVU with permission from the rights-holder(s). You are free to use this Thesis in any way that is permitted by the copyright and related rights legislation that applies to your use. For other uses you must obtain permission from the rights-holder(s) directly, unless additional rights are indicated by a Creative Commons license in the record and/ or on the work itself. This Thesis has been accepted for inclusion in WVU Graduate Theses, Dissertations, and Problem Reports collection by an authorized administrator of The Research Repository @ WVU. For more information, please contact researchrepository@mail.wvu.edu. 
Serotonergic Modulation of Inhibitory Input to Lateral Horn Affects Odor-Mediated Attraction in D. melanogaster

Ayad Auda

Thesis submitted to the Eberly College of Arts and Sciences

at West Virginia University

in partial fulfillment of the requirements

for the degree of

Master of Science

in

Biology

Andrew M. Dacks, Ph.D., Chair

Sarah M. Farris, Ph.D.

Kevin C. Daly, Ph.D.

Department of Biology

Morgantown, WV

2017

Keywords: Neuromodulation, Serotonin, Olfaction, Behavior, Drosophila

Copyright 2017 Ayad Auda 


\section{Abstract \\ Serotonergic Modulation of Inhibitory Input to Lateral Horn Affects Odor-Mediated Attraction in D. melanogaster}

\section{Ayad Auda}

To survive, animals must eat, mate, and avoid danger by sensing external stimuli, processing sensory information into perception, and eliciting the appropriate behavioral responses in accordance with their physiological states. Unlike learned responses, innate responses are genetically inherited and do not require the animal to have a previous encounter with the stimulus to elicit an action. However, innate responses can be flexible based on the physiological state of the animal. This flexibility is achieved through constant alterations in network dynamics which ensures that the animal's responses are most effective. This alteration occurs to some extent by modulatory neurons/nuclei at multiple processing stages of a neural network and mediated via several modulatory receptor subtypes in the network. In this study, I take advantage of the well-characterized olfactory system of Drosophila to explore connectivity between a single pair of modulatory serotonergic neurons, the CSD neurons, and a population of ventral projection neurons, vPNs. Both types of neurons span two different olfactory processing stages and vPNs are vital for eliciting attraction to odors that have innately important values. I further, investigate the degree to which innate responses can be modulated by dissecting the role of one serotonergic receptor, 5-HT1A, expressed by vPNs in innate attraction and the behavioral consequences of reducing its expression in vPNs. I found that CSD neurons synapse upon the dendrites and the axons of vPNs in the antennal lobe and lateral horn, respectively, to potentially modulate the activities of vPNs in both regions simultaneously. I also found that reducing the expression of the 5-HT1A receptor in vPNs selectively increases flies' attraction to the innately attractive odor apple cider vinegar, but neither to other food odors with different biological values, nor to aversive odors such as benzaldehyde. This study contributes to our understanding of the simultaneous modulation of interconnected networks by a single pair of modulatory neurons as well as the consequences of neuron class-specific expression of a single modulatory receptor. The outcome knowledge is critical for better understanding of neural coding processes in sensory systems and the extent to which innate behaviors are modulated. 


\section{ACKNOWLEDGMENTS}

First, I would like to thank my adviser Dr. Andrew Dacks for the great support and encouragement he has provided me throughout my Master's program. Without his continuous help and guidance, this work would have never been accomplished. There are literally not enough words to explain how grateful I am and how lucky I feel to have had the opportunity to work in his lab. It was a great pleasure and honor to be one of his students.

Second, I would also like to thank my committee members, Dr. Kevin Daly and Dr. Sarah Farris, who kept their doors open for me all the time and have always been there for me as mentors and friends.

I would also like to say thanks to my father and my sister and her new wonderful family. They have never lost faith in me and always supported me with every small step I made towards this degree, even though they were thousands of miles away.

Finally, I would like to thank my lab colleagues who were great friends and company especially Kaylynn Coates for the tremendous help in all aspects. 


\section{TABLE OF CONTENTS}

ABSTRACT .ii

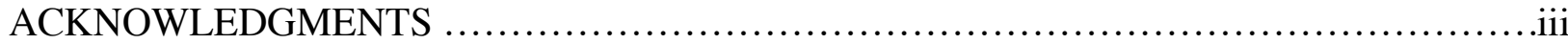

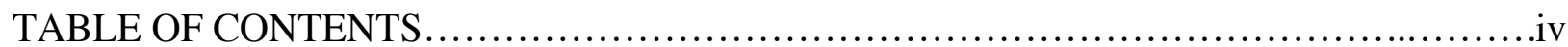

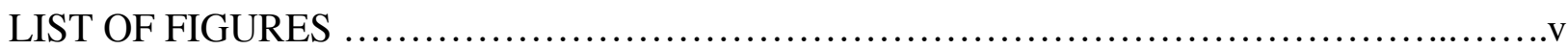

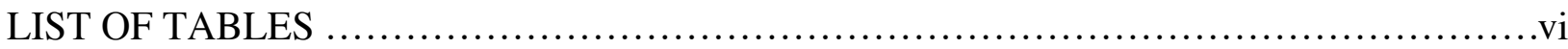

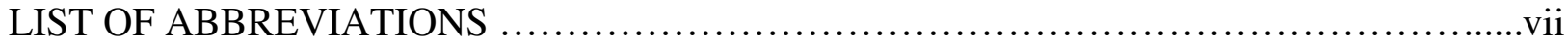

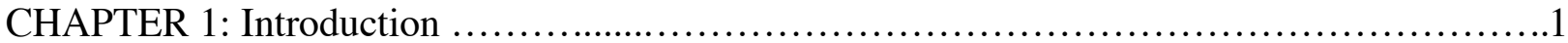

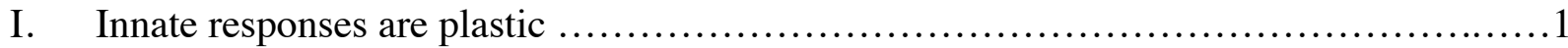

II. Types of inter-neural communications ........................................

III. $\quad 5$-HT receptors expressed in the brain of Drosophila .............................2

IV. Olfactory system in Drosophila ............................................

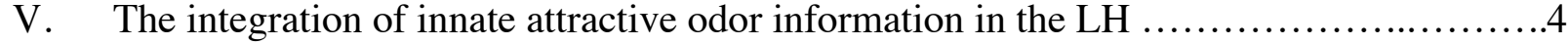

CHAPTER 2: Determining the Synaptic Interaction Between vPNs and CSD Neurons ..........6

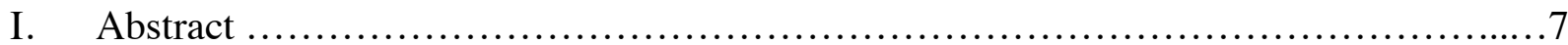

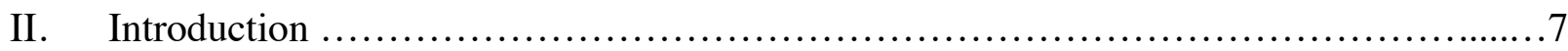

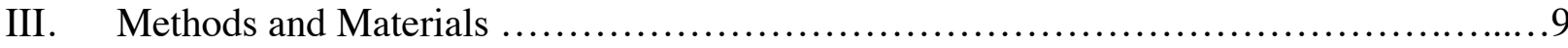

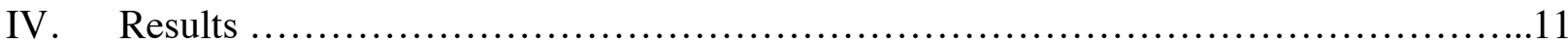

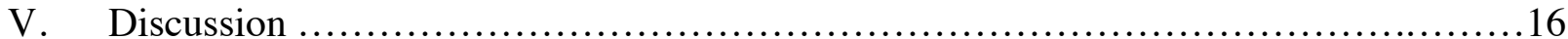

CHAPTER 3: Determine the Contribution of 5-HT1A Receptor Expression by vPNs on

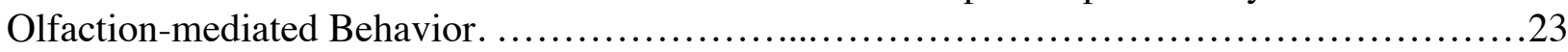

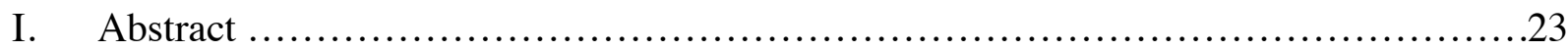

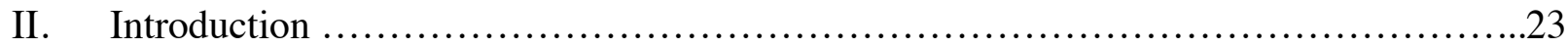

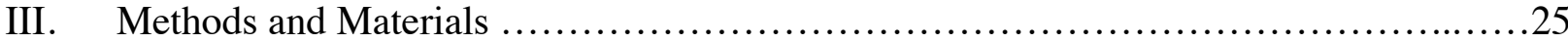

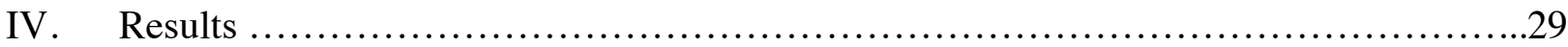

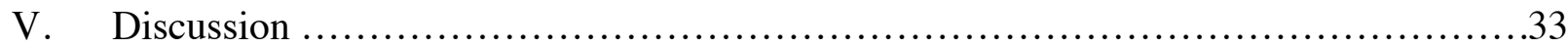

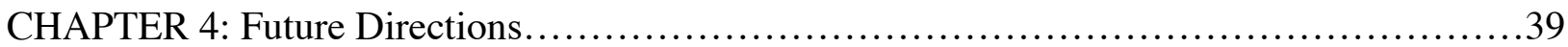

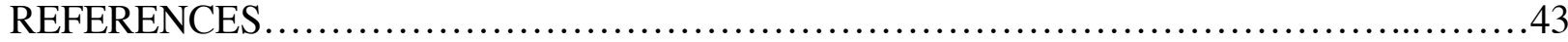




\section{LIST OF FIGURES}

Figure 1. Olfactory circuitry of D. melanogaster \& the morphology of CSD neurons ...........6

Figure 2. Morphology of the vPNs-LexA lines ............................................

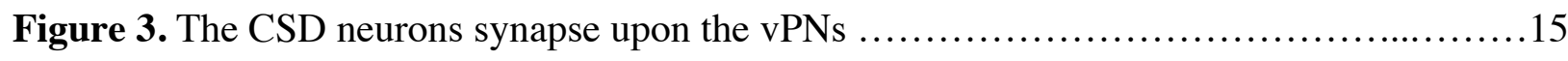

Figure 4. LH circuitries suggested by Parnas et al, (2013) and Liang et al, (2013) .............20

Figure 5. Knockdown of 5-HT1A expression in vPNs changes flies' responses to ACV but neither to BZE nor to banana extract ............................................................ 


\section{LIST OF TABLES}

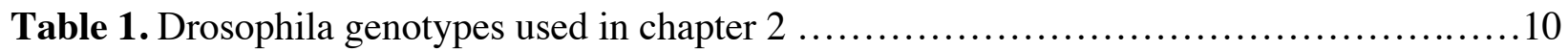

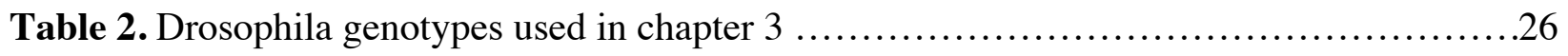

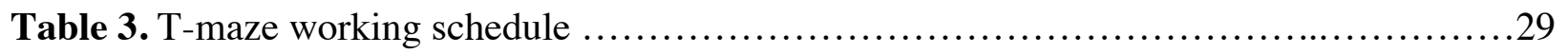




\section{LIST OF ABBREVIATIONS}

ORNs Olfactory receptor neurons

LNs Local interneurons

ePNs Excitatory projection neurons

vPNs Ventral projection neurons

adPN Antero-dorsal projection neurons

IPN Lateral projection neurons

vlPrNs Ventrolateral protocerebral neurons

CSDs Contralaterally projecting, serotonin-immunoreactive, deutocerebral neurons

mlALT Mediolateral antennal lobe tract

mALT Medial antennal lobe tract

1ALT Lateral antennal lobe tract

GFP Green fluorescent protein

GRASP GFP reconstitution across synaptic partner

Nrx Neurexin

ACV Apple cider vinegar

BV Balsamic vinegar

BZE Benzaldehyde

BE Banana extract 
cVA Cis-vaccenyl acetate

PLC Phospholipase C

PLK Protein Kinase C

DAG Diacylglycerol

IP3 Inositol-3-phosphate

EPSPs Excitatory post synaptic potentials 


\section{Chapter 1: Introduction}

\section{Innate responses are plastic}

In a continuously-changing environment, it is crucial for animals to constantly adapt by generating the appropriate behavioral responses that ensure their survival. These responses are usually achieved when sensory information received by peripheral sensory organs are conveyed and integrated in specialized regions in the brain. Generally, behavioral responses can be categorized into two types, learned and innate responses. Unlike learned responses which require a prior experience, innate responses provide naive animals with the ability to survive by responding to important environmental stimuli. Though innate responses are crucial, they are not rigid and are vulnerable to modification so that the generated behavior would occur in the right context to best fit the physiological state of the animal and in perfect correspondence to external stimuli. This plasticity of innate responses, to a certain degree, is achieved via a process called neuromodulation that could potentially occur at any of the processing stages of neural information pathways.

\section{Types of inter-neural communications}

Neurotransmission is the process by which one neuron, fast and chemically, conveys information to another single neuron causing it to be either excited or inhibited (Katz, 1999). This form of "talk" between neurons is considered as the primary means of the neuronal communication (Katz, 1999). Quite the contrary, neuromodulation is a slower type of communication that is achieved when a modulatory neuron releases signaling chemicals to provoke similar and/or diverse effects on targeted neurons without necessary eliciting an action potential in them (Kupfermann, 1979) or changing their spontaneous activity (Dacks et al., 2008). Neuromodulation provides neural networks with flexibility by altering the biophysical properties and/or synaptic efficiency of neurons. Neuromodulators change the excitability of a neuron by binding to a G Protein Coupled Receptor (GPCR) to activate second messenger cascades, which alter the conductance of membrane channels. For instance, in moths, serotonin (5-HT) decreases two $\mathrm{K}^{+}$conductance of antennal lobe (AL) neurons (Kloppenburg et al., 1999; Mercer et al., 1995) causing them to be more resistant. The increase in resistance leads to the neurons to be more sensitive, and thus, responsive to subthreshold stimuli (Kloppenburg et al., 
1999; Dacks et al., 2008; Dacks et al., 2009). Synaptic efficiency, on the other hand, is modified when a neuromodulator facilitates/inhibits the release of neurotransmitter from the vesicles into the synaptic cleft. For instance, in the swim circuit of the marine mollusk Tritonia diomedea, a subset of serotonergic neurons, called the dorsal swim interneurons (DSIs), enhance the neurotransmitter release of the $\mathrm{C} 2$ interneuron, and subsequently the excitatory post synaptic potentials evoked by C2 in its targeted neuron, DFN (Katz and Frost, 1995). The overall consequence is changing the motor cell responses, and consequently, shaping the behavioral responses to different environmental stimuli.

\section{5-HT receptors expressed in the brain of Drosophila}

Serotonin (5-hydroxytryptamine: 5-HT) regulates several behavioral and physiological processes in animals. It does so by functioning as a signaling chemical and eliciting its effects on distinctive types of receptors. For instance, serotonergic signaling plays an important role in controlling and regulating mood and sleep in mammals (Weiger, 1997; Blenau and Thamm, 2011). In vertebrates, 5-HT receptors are classified into seven different classes. Each of these classes is further sub-categorized into subclasses based on their pharmacological properties (Nichols and Nichols, 2008). The diversity of 5-HT receptors makes the task of studying the functional contribution of each receptor to the overall effects of 5-HT extremely challenging. Thus, the use of a simpler system with fewer components such as the olfactory system of Drosophila is considered useful since it is easier to perform direct experiments in it. In adult Drosophila, two contralaterally projecting, serotonin-immunoreactive, deutocerebral (CSD) neurons project to several brain regions and are the sole source of endogenous 5-HT in the olfactory system of the fly (Kent et al., 1987; Dacks et al., 2006; Coates et al., in review). Serotonergic effects elicited by CSD neurons are mediated by five orthologs of the human 5-HT receptors, these are; 5-HT1A, -1B, -2A, -2B and -7 receptor types (Nichols and Nichols, 2008; Blenau and Thamm, 2011), which have been identified as GPCRs suggesting that 5-HT functions only as neuromodulator in Drosophila (Blenau and Thamm, 2011). For instance, exogenous 5-HT enhances the strength of the odor-evoked responses of ePNs (Dacks et al, 2009). Furthermore, these receptor types are coupled to different G-protein alpha subunits; thus, they elicit different neuromodulatory effects on target neurons. For example, the 5-HT1A receptor type is coupled to $G_{i}$ alpha subunit, which is responsible for inhibiting the synthesis of 
cAMP in the cell, frequently eliciting an inhibitory response (Witz et al., 1990; Nichols and Nichols, 2008).

\section{Olfactory system in Drosophila}

Flies detect odors with olfactory receptor neurons (ORNs) contained in the sensilla of the antennas and maxillary palps (Vosshall et al, 2000; $\mathrm{Ng}$ et al., 2002). The sensilla of an individual antenna collectively house $\sim 1200$ ORNs, while a single maxillary palp's sensilla house $\sim 120$ ORNs (Laissue and Vosshall, 2008). ORNs send their axons to the antennal lobe (AL), the first olfactory processing center within the fly's brain and the analog of the mammalian olfactory bulb (Vosshall et al, 2000; Ng et al., 2002; Stocker et al., 2009). In general, neurons expressing the same olfactory receptors converge on 1 or few glomeruli of the 53 comprising the AL, (Vosshall et al., 2000; Laissue and Vosshall, 2008).

Within the AL, ORNs synapse upon second-order neurons called the projection neurons (PNs) (Fig. 1A). There are roughly 150 PNs extending dendrites to AL glomeruli (Okada et al., 2009), and PNs convey olfactory information to downstream centers such as the mushroom body (MB) and the lateral horn (LH), two second-order olfactory centers known for their role in olfactory learning and memory (Heisenberg, 2003; Davis, 2005; Liang et al., 2013), and odorevoked innate behavior (Heimbeck et al., 2001), respectively. The cell bodies of the PNs are distributed in three different clusters surrounding the AL, these are; the ventral, antero-dorsal, and lateral cell clusters. Both the antero-dorsal PNs (adPNs) and lateral PNs (IPNs) are cholinergic (Okada et al., 2009) (Fig. 1A). They are referred to as excitatory PNs (ePNs) and they project to both the MB and LH. In addition to ePNs, there are $\sim 51$ GABAergic cells in the ventral cluster named the ventral projection neurons (vPNs) and have been characterized using the two GAL4 lines, GH146 and Mz699 (Ito et al., 2007; Wilson and Laurent, 2005; Okada et al., 2009; Parnas et al., 2013). Mz699-GAL4 line labels 45 neurons, while GH146-Gal4 labels the rest of the cells ( 6/51) (Okada et al., 2009; Wilson and Laurent, 2005; Liang et al., 2013). Based on their transmitter content and morphology, it is evident that ePNs and vPNs serve different roles within the olfactory system. For instance, ePNs are uniglomerular (they individually innervate one glomerulus) (Liang et al., 2013) and send excitatory information to the MB and LH, whereas the vast majority of vPNs are multi-glomerular (send dendrites to $\sim 5$ glomeruli each) and relay inhibitory input into the LH directly, bypassing the MB (Okada et al., 
2009). Furthermore, the density of Mz699-labeled vPN innervations is not homogenous between glomeruli (e.g. DM2 and DM5 have dense innervation, while DL4\& DL5 have light innervation) (Strutz et al., 2014). Together, this suggests that ePNs and vPNs differ in terms of the type of odor information they integrate, as well as the type of input they provide to higher brain regions (Wang et., 2003; Wilson et al., 2004; Silbering et al., 2008; Liang et al., 2013; Strutz et al., 2014)

\section{The integration of innate attractive odor information in the LH}

A growing body of work has demonstrated that $\mathrm{LH}$ directs odor-mediated innate responses of the fly. That is, a decision as to whether an odor is aversive or attractive is mainly made when odor signals are processed in the LH. The direct axonal projection of vPNs to the LH implies that they likely have a role in directing the odor-guided innate behavior of the flies. For instance, Parnas et al., 2013 argue that vPNs have a key role in improving the ability of the fly to distinguish closely related innately attractive food odors. This improvement, they claim, is achieved via pre-synaptic inhibition applied by vPNs on the axon terminals of ePNs in the LH, which would enhance the ability to discriminate odors by suppressing weaker odor responses to produce a sharper neural representation. Another proposed role of vPNs is that they selectively suppress the activation of repulsion by inhibiting a group of third-order neurons named the ventro-lateral projection neurons (vlPrNs) (Liang et al., 2013) to elicit attraction to innately attractive odors (Strutz et al., 2014). Overall, previous observations imply that vPNs have a key role in shaping the flies' innate responses to odors that have innately important values.

5-HT is widely distributed neuromodulator that provides flexibility to sensory networks both in mammals (Nichols and Nichols, 2008; Petzold et al., 2009; Huang et al., 2017) and insects (Dacks et al., 2008; Dacks et al., 2009; Zhang and Gaudry, 2016). For instance, in Drosophila, 5-HT has a key role in changing the odor-evoked response of AL glomeruli (Dacks et al., 2009; Zhang and Gaudry, 2016). But how does endogenous 5-HT release in the olfactory system contribute to the modulation of flies' responses to odors that have innately important values? Is this modulation occurring at one or multiple processing stages? vPNs as a population express all 5-HT receptor types (Sizemore and Dacks, 2016) and send exclusive processes to the $\mathrm{AL}$ and LH where CSD neurons also have processes, suggesting a potential modulatory role for endogenous 5-HT released by CSD neurons on vPNs in either or both processing stages. Considering the role of vPNs in innate attraction, such modulation would also potentially affect 
flies' responses to innately attractive odors. While it is known that serotonin modulates odor responses in the AL (Dacks et al., 2009; Zhang and Gaudry, 2016), the contribution of each serotonergic receptor type to the overall modulatory effect of serotonin, and subsequently, to innate odor-guided behavior in Drosophila, has never been examined. In this study, I used two different behavioral assays in conjunction with anatomical methods and genetic techniques to investigated the synaptic connectivity of CSD neurons and vPNs as well as the contribution of 5HT1A-mediated serotonergic neuromodulation to the perception of odor valence in the olfactory system of Drosophila. I found that CSD neurons synapse upon vPNs in two different processing stages, AL and LH, suggesting that vPNs receive simultaneous axo-dendritic and axo-axonic serotonergic input from CSD neurons in in the AL and LH, respectively. Further, I found that reducing 5-HT1A-mediated inhibition of vPNs increases the flies' attraction to the naturally important odors apple cider vinegar (ACV) as well as balsamic vinegar (BV), but doesn't affect their response to the aversive odor benzaldehyde (BZE). This suggests a selective, 5-HT1Amediated modulatory role of endogenous 5-HT on flies' attraction towards odor carrying specific biological values. The outcome knowledge of this study is critical for better understanding of the serotonergic modulation of neural coding processes in the olfactory system of Drosophila and the consequences of this modulation on odor-driven innate behavior. 


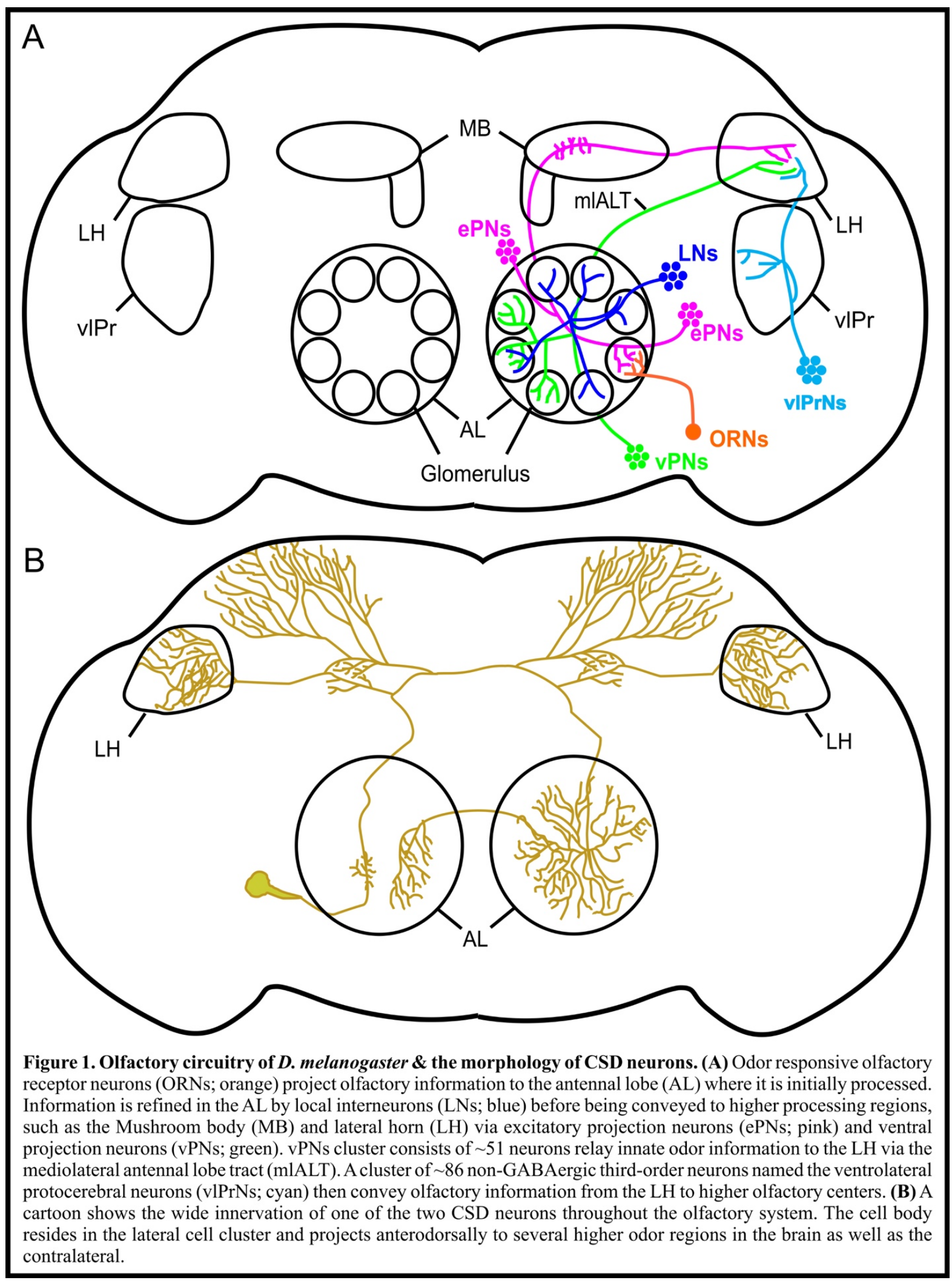




\section{Chapter 2: Determining the Synatic Interaction Between vPNs and CSD Neurons.}

\section{Abstract}

An organism must constantly adjust its behavior based on the continuously changing environment in which it's immersed to ensure its survival. One way this is achieved is by the release of neuromodulators by modulatory networks. A modulatory neuron or nucleus can simultaneously affect multiple processing stages of a neural network. Due to the complexity of modulatory networks, the consequences of such multi-layer modulation, even in relatively simple neural networks, are not well understood. For instance, in the olfactory circuit of Drosophila, synaptic 5-HT is released by CSDs neurons in the AL and LH suggesting modulatory effects in these centers. Furthermore, vPNs, which express all 5-HT receptor types encoded in the fly's genome, also send processes to AL and LH. Collectively, this suggests that the CSD neurons are modulating the vPNs through the release of serotonin. Yet, it is unknown in which of the two olfactory centers, AL or LH, CSD neurons synapse upon vPNs, and if a modulatory effect is elicited directly or indirectly by CSD neurons on vPNs. In this chapter, I determine that CSD neurons synapse on vPNs in the AL, and to a lesser extent, in the LH. My data implies that the CSD neurons modulate olfactory processing simultaneously in two processing stages, AL and LH, by targeting both the dendrites and axon terminals of vPNs in these areas.

\section{Introduction}

Neuromodulatory systems play crucial roles in modifying fundamental behavior in response to their changing environment and internal physiological states. These systems typically send projections that span several processing stages and are known to be extremely complex due to the extensive dynamic interactions between them. For instance, in mammals, serotonergic neurons originated within the Raphe nuclei project to several cortical and subcortical brain areas to modulate their activities (Hurley et al., 2004; Jacobs and Azmitia, 1992). Among these regions are the frontal cortex, a brain region known for its role in memory, language and complex behaviors (Brower and Price, 2001), and the amygdala, a part of the limbic system responsible for the perception of emotions and the control of aggression (Bzdok et al., 2012). 
Simultaneously, however, the frontal cortex and amygdala receive input from multiple other modulatory entities such as ventral tegmental area (Aransay et al., 2015) and the locus coeruleus (Strawn and Geracioti, 2008). Moreover, studies have shown neural connections between the amygdala and frontal cortex. The simultaneous targeting of frontal cortex and amygdala by several modulatory entities as well as the dynamic interconnections between all previous modulatory nuclei, therefore, make it harder for us to investigate the consequences of modulating each of two interconnected networks that simultaneously receive inputs from the same modulatory source.

To study the consequences of simultaneously modulating two interconnected networks, I used a simpler system, the well-characterized olfactory system of Drosophila melanogaster. Olfaction in the vinegar fly starts when ORNs detect odors and send information to the AL for a primary processing. In the AL, the ORNs synapse on the second order projection neurons (PNs) which convey olfactory signals to downstream olfactory networks, such as the later horn LH, a well-studied higher olfactory center known for its role in directing innate responses to odors (Fig. 1A). vPNs were obvious, great candidates because they collectively express all 5-HT receptor types. Moreover, this population sends dendrites to the AL and exclusive projections to the LH, bypassing other olfactory centers. Thus, vPNs and CSD neurons both innervate two anatomically-separate levels of the olfactory network, which makes them an ideal model for studying the simultaneous modulation of a population of neurons in multiple processing stages (Fig. 1A and 1B). There are $\sim 51 \mathrm{vPN}$ cell bodies that are located ventral to the AL. Out of the 51 vPNs, 6 are labeled by the GH146-Gal4 line (Stocker et al., 1997) and found to be GABAergic (Wilson and Laurent, 2005; Jefferis et al., 2007; Liang et al., 2013). The other 45 cells are labeled by the Gal4 line Mz699 (Lai et al., 2008; Okada, et al., 2009). Out of the 45 neurons, 39 show multi-glomerular innervation in the AL and reported to be GABAergic (Okada et al., 2009; Strutz et al., 2014). The other 6 were found to be uni-glomerular and express the neurotransmitter acetylcholine (Wilson and Laurent, 2005; Liang et al., 2013; Strutz et al., 2014). Several studies have suggested a vital role for vPNs in coding and integrating odor features in the olfactory circuitry of fruit flies (Strutz et al., 2014). However, to my knowledge, no study has looked at the modulatory effects of endogenous 5-HT on vPNs' role in odor-guided behavior. CSD neurons are the sole source of synaptic 5-HT in the fly's olfactory centers (Kent et al., 1987; Dacks et al., 2006; Coates et al., in revision). They have a broad innervation pattern 
demonstrated by sending processes to both the primary olfactory center, AL, and the downstream center, LH, among other olfactory centers (Dacks et al., 2006) (Fig. 1B). They are also known for playing a crucial role in modulating olfactory processes in fly brain (Dacks et al, 2009; Zhang and Gaudry, 2016). Using a protein trap technique in which Gal4 is inserted to the coding intron of each of the 5 serotonin receptors (Gnerer, et al., 2015), Sizemore and Dacks, (2016) showed that vPNs as a population express all 5 types of 5-HT receptors subjecting them to a potential serotonergic modulation. Additionally, by driving synaptotagmin.eGFP (Zhang et al., 2002) in CSD neurons, (Coates et al, in revision) showed GFP puncta expressed by the CSD neurons in both the AL and $\mathrm{LH}$, indicating that CSD neurons potentially have synaptic output in both olfactory centers. Based on these observations, a couple of questions could be asked; Do vPNs receive synaptic input from the CSD neurons? If so, at which of the two olfactory centers do the CSD neurons synapse onto vPNs? Do the CSD neurons modulate both the input and output domains of vPNs at different locations? We therefore used the GFP Reconstitution Across Synaptic Partners (GRASP) to demonstrate that vPNs receive input from the CSD neurons mainly in the AL and to a lesser degree in the LH.

\section{Materials \&Methods}

Fly stocks: Flies were maintained on Nutri-Fly "German Food" Sick Fly Formulation at 50\% humidity and $25^{\circ} \mathrm{C}$, on a $12: 12$ dark-light cycle. The following fly stocks were used: $U A S-R F P$; LexAop-GFP (BDSC \#32229), UAS-GFP (BDSC \#32185), R60F02-Gal4 (BDSC \#48228), LexAop-spGFP11::CD4;UAS-spGFP1-10::Nrx (provided by Dr. Nirao Shah), MB465C splitGal4 (Aso et al., 2014), LexAop-GFP (BDSC \#32207), GMR76H03-LexA (BDSC \#54954), GMR37E10-LexA (BDSC \#54200), T(2;3)ap[Xa], ap[Xa]/CyO; TM3, Sb (BDSC \#2475). 
Table 1. Drosophila genotypes used in chapter 2

\begin{tabular}{|l|l|}
\hline \multicolumn{1}{|c|}{ Figure \# } & \multicolumn{1}{c|}{ Genotypes (Transgenes with Bloomington number) } \\
\hline Figure $2 \mathrm{~A}_{1-3} \& 2 \mathrm{~B}_{1-2}$ & GMR76H03-LexA (BDSC \#54954) \\
\hline Figure $2 \mathrm{C}_{1-3} \& 2 \mathrm{D}_{1-2}$ & GMR37E10-LexA (BDSC \#54200) \\
\hline Figure 3A, 3B, \& 3D & R76H03-LexA/UAS-RFP;MB465c split-Gal4/LexAop-GFP \\
\hline Figure 3C, 3E & $\begin{array}{l}\text { R76H03-LexA/LexAop-spGFP11::CD4; MB465c split-Gal4/ UAS- } \\
\text { spGFP1-10:NNx }\end{array}$ \\
\hline Figure 3F, 3G, \& 3I & R37E10-LexA/UAS-RFP;MB465c split-Gal4/LexAop-GFP \\
\hline Figure 3H, 3J & $\begin{array}{l}\text { R37E10-LexA/LexAop-spGFP11::CD4; MB465c split-Gal4/UAS- } \\
\text { spGFP1-10::Nrx }\end{array}$ \\
\hline
\end{tabular}

Antibodies: The following primary antibodies and dilutions were used for neuroanatomical characterization: 1:1,000 Chicken anti-GFP (Abcam, \#ab13970), 1:5,000 Rabbit anti-5-HT (Immunostar, \#20079), 1:250 Rabbit anti-DsRed (Clontech \#632496), and 1:50 Mouse antiBruchpilot (Developmental Studies Hybridoma bank: mAbnc82). Mouse anti-GFP (Sigma \#G6539) was used in GRASP protocols as it only recognizes the reconstituted GFP (Gordon and Scott, 2008) Because this antibody is raised in mouse and so is the commonly used neuropil antibody, anti-Bruchpilot nc82, subsequent images of GRASP immunoreactivity are shown without a neuropil label (Wagh et al., 2006). The following secondary antibodies were used at a 1:1000 dilution: Donkey anti-Mouse 488, Donkey anti-Rabbit 488, Donkey anti-Rabbit 546, Donkey anti Goat-546, Goat anti Mouse-633.

Stable lines: The stable line GMR76H03-LexA;GMR-MB465CspGal4 was established by crossing female virgins from each of GMR76H03-LexA and MB465C split-Gal4 lines individually with the balancer line 2475 (T(2;3)ap[Xa], ap[Xa]/CyO; TM3, Sb). F1 progenies from both crosses with specific traits (to ensure that no recombination processes across the associated chromosomes flies had occurred) were then collected and self-crossed. Specifically, flies bearing the genotypes (vPN-LexA/CyO;III/TM3) \& (II/CyO;MB465C/TM3) were selfcrossed. Finally, progenies bearing the genotypes (vPN-LexA/vPN-LexA;III/TM3) \& (II/CyO;MB465C/MB465C) from both crosses were collected and crossed with each other to 
generate the final stable line (vPN-LexA/CyO;MB465C/TM3). The second stable line, GMR37E10-LexA;GMR-MB465CspGal4 was made using the same method described above.

Immunocytochemistry: Brains were dissected in Drosophila external saline and fixed in $4 \%$ paraformaldehyde for 30-45 minutes on ice and then washed (4 times, 15 minutes each) in PBST (Phosphate Buffered Saline with 0.5\% Triton-X100). To minimize tracheal artifacts, brain were then treated with an ascending-descending ethanol series $(30 \%, 50 \%, 70 \%, 90 \%, 100 \%$ 3xs, $90 \%, 70 \%, 50 \%, 30 \%$ ), with each wash lasting 5 minutes. Brains were then blocked for 60 minutes in 2\% BSA (Bovine Serum Albumin) (Jackson ImmunoResearch; \#001-000-162) in PBST. Brains were then incubated in a blocking solution containing 5mM sodium azide and diluted primary antibodies at $4^{\circ} \mathrm{C}$. After incubation, brains were washed, blocked (same as previous steps), and incubated in secondary antibodies diluted in block solution with $5 \mathrm{mM}$ sodium azide at $4^{\circ} \mathrm{C}$ for 24 hours. Brains were then washed in PBST (2 times, 15 minutes each) and PBS (2 times, 15 minutes each), run through an ascending glycerol series $(40 \%, 60 \%, \&$ 80\%), and mounted in VectaShield (Vector Labs Burlingame, CA \#H-1000). Brains were scanned using a Olympus confocal microscope FV1000 (20x, 40x or 60x oil immersion lens). All Images were acquired using Olympus FluoView software, and processed using CorelDraw X4. To test if the CSD neurons are anatomically presynaptic to vPNs, each stable line was crossed with LexAop-spGFP11::CD4;UAS-spGFP1-10::Nrx, and brains of F1 progeny were then dissected and run through standard immunocytochemistry protocols (see below) before being scanned for GRASP signals.

\section{Results}

To investigate the anatomical overlap of CSD and vPNs projections in the primary (AL) and secondary (LH) olfactory processing regions, I first used the FlyLight Gal4 database (http://flIb.janelia.org/cgi-bin/flew.cgi), to identify LexA lines in which the vPNs are expressed. The two lines that I chose to use are R76H03-LexA and R37E10-LexA (Fig. 2). In R76H03LexA, vPNs are the only neurons expressed in either the AL or the LH (Fig. $2 \mathrm{~A}_{1-3}$ ). In R37E10LexA, the vPNs are the only neurons expressed in the AL, though, a cluster of LH-LNs was also observed within the $\mathrm{LH}$ in addition to the vPNs (Fig. $2 \mathrm{C}_{1-3}$ ). R76H03-LexA is expressed by 12-15 neurons of the $\sim 51$ vPNs, while the other line, R37E10-LexA, labels 8-12 vPNs. Moreover, the two lines drive LexA expression in several glomeruli in the ALs. R76H03-LexA occupies 17 


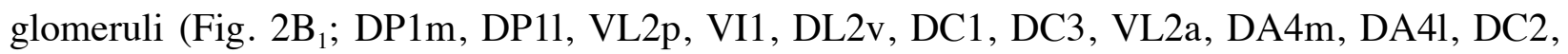
VM2, DM1, DL2d, VA1d, VA6, and DA2) and R37E10-LexA occupies 9 glomeruli (Fig. 2D VA4, DA1, VA11m, VA2, DM1, DL2d, VA1d, VA6, and DA2). Thus, the two LexA lines only overlap in DM1, DL2d, VA1d, VA6, and DA2. In both lines, I observed that vPNs seem to differ in the width of their innervations. For instance, in some cases a glomerulus would receive a very sparse innervation, while in other cases, a glomerulus might receive a dense innervation (Fig. $\left.2 \mathrm{~B}_{2} \& 2 \mathrm{D}_{2}\right)$.

Data from our lab using DsCam-GFP, a dendritic marker found in developing neurons (Wang et al., 2002), shows that vPNs receive synaptic input exclusively in the AL, which is consistent with previous studies on the vPNs (Ito et al., 1997: Parnas et al., 2013). To investigate the olfactory center at which vPNs receive synaptic input form CSD neurons, I used $R 76 H 03$ and R37E10-LexAs which drive expression in the vPNs, and MB465c split-Gal4 which is expressed only in the CSDs to generate stable lines for testing GRASP (see methods). The two stable lines had the genotypes R76H03-LexA;MB465c split-Gal4 \& R37E10-LexA;MB465c split-Gal4. This enabled us to use two binary systems (Gal4 \& LexA) to simultaneously drive the expression of the two transgenes UAS and LexAop in the two populations of neurons. Next, I crossed each of the stable lines individually to a reporter line UAS-RFP;LexAop-GFP, so that the expression of RFP and GFP was driven in CSD neurons and vPNs, respectively. I observed clear physical overlap of the vPNs and CSD neurons in both the AL (Fig. 3D \& 3I) and the LH (Fig. 3B \& 3G), suggesting potential connectivity between the two populations. 


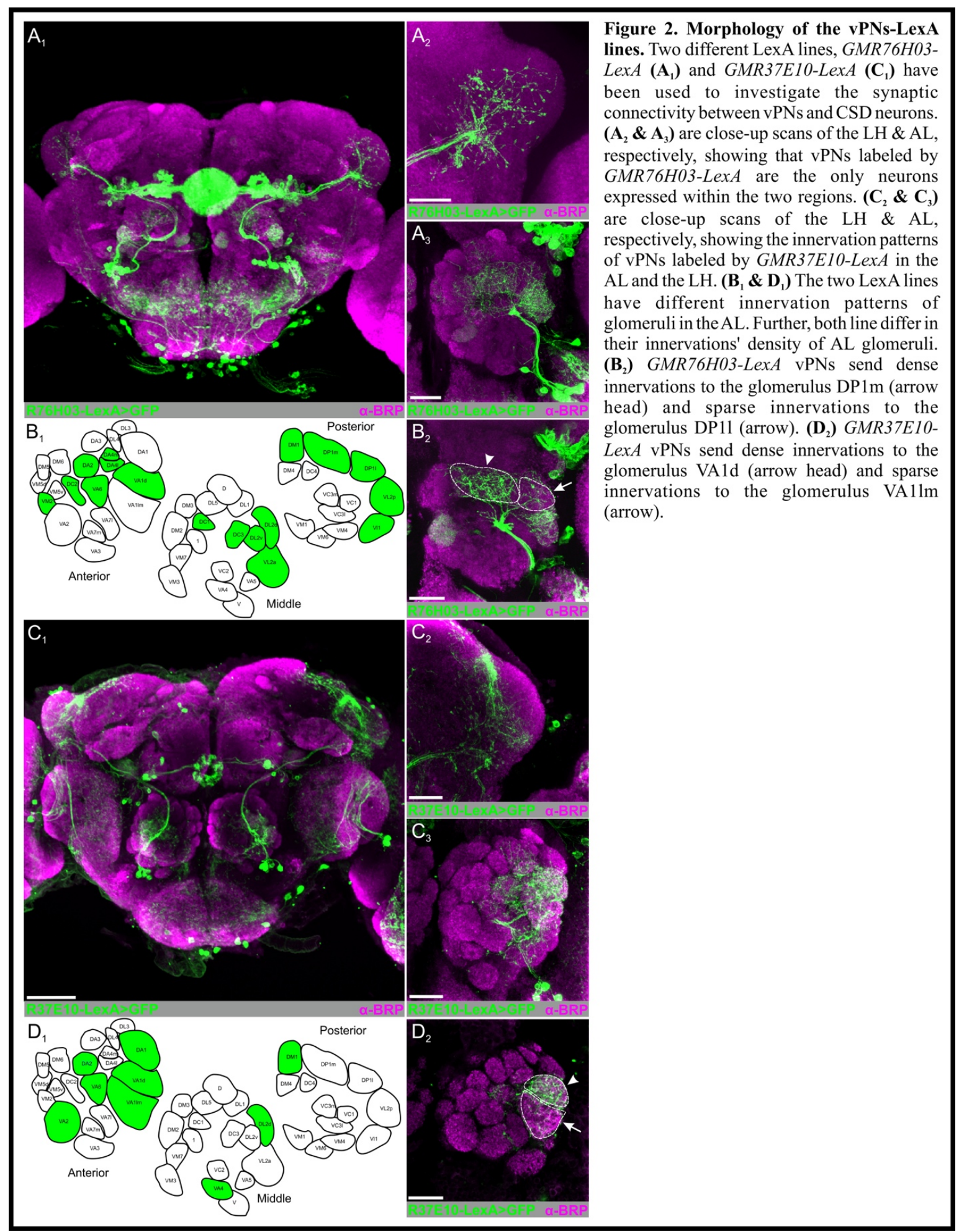


Next, I used GFP Reconstitution Across Synaptic Partners (GRASP) (Feinberg et al., 2008) to investigate the synaptic connectivity between vPNs and CSD neurons. In this technique, the expression of a membrane bound GFP fragment is driven by Gal4 expressing neurons, while the other membrane bound GFP fragment is expressed in different, LexA-expressing neurons. If two neurons are in close physical proximity to each other, the two fragments reconstitute and a functional GFP is formed. The formation of a complete and functional GFP indicates that the two neurons are likely contacting each other. To investigate whether CSD neurons and vPNs are actually synapsing upon each other and the directional flow of the neural signal (i.e. whether the CSD neurons are synapsing onto vPNs), I used Neurexin GRASP (LexAopspGFP11::CD4;UAS-spGFP1-10::Nrx) (Fan et al., 2013). Neurexin is a presynaptic, transmembrane protein important for the appropriate development and formation of synapses. Specifically, neurexin binds to a postsynaptic protein named neuroligin to physically connect neurons to each other (Knight et al., 2011). In this UAS-line, a fragment of GFP is inserted into the gene coding region for the presynaptic protein neurexin, which allows the GFP to be constrained to the presynaptic regions of the Gal4-expressing neurons but not LexA expressing neurons (i.e. to CSD neurons but not vPNs), which avoids any potential artifact due to the neurons being in close physical proximity, and thus touching, but not synapsing onto each other. By crossing this line to R76H03-LexA;MB465c split-Gal4 line, I observed strong GRASP signal distributed throughout the AL (Fig. 3E). I also observed sparse GRASP signal in the LH (Fig. 3C). Likewise, using the second stable line R37E10-LexA;MB465c split-Gal4 I also observed strong GRASP signal throughout the AL (Fig. 3J) and sparse GRASP signal in the LH (Fig. 3H). Using an activity-dependent form of GRASP (synaptobrevin GRASP), our lab also demonstrated that the CSDs synapse onto R76H03 vPNs (Coates personal communication) which validates both the GRASP technique and my results. Together, based on the data I provide above, I demonstrate that CSD neurons synapse onto the dendrites and axons of vPNs in two processing stages, AL and LH, respectively. 


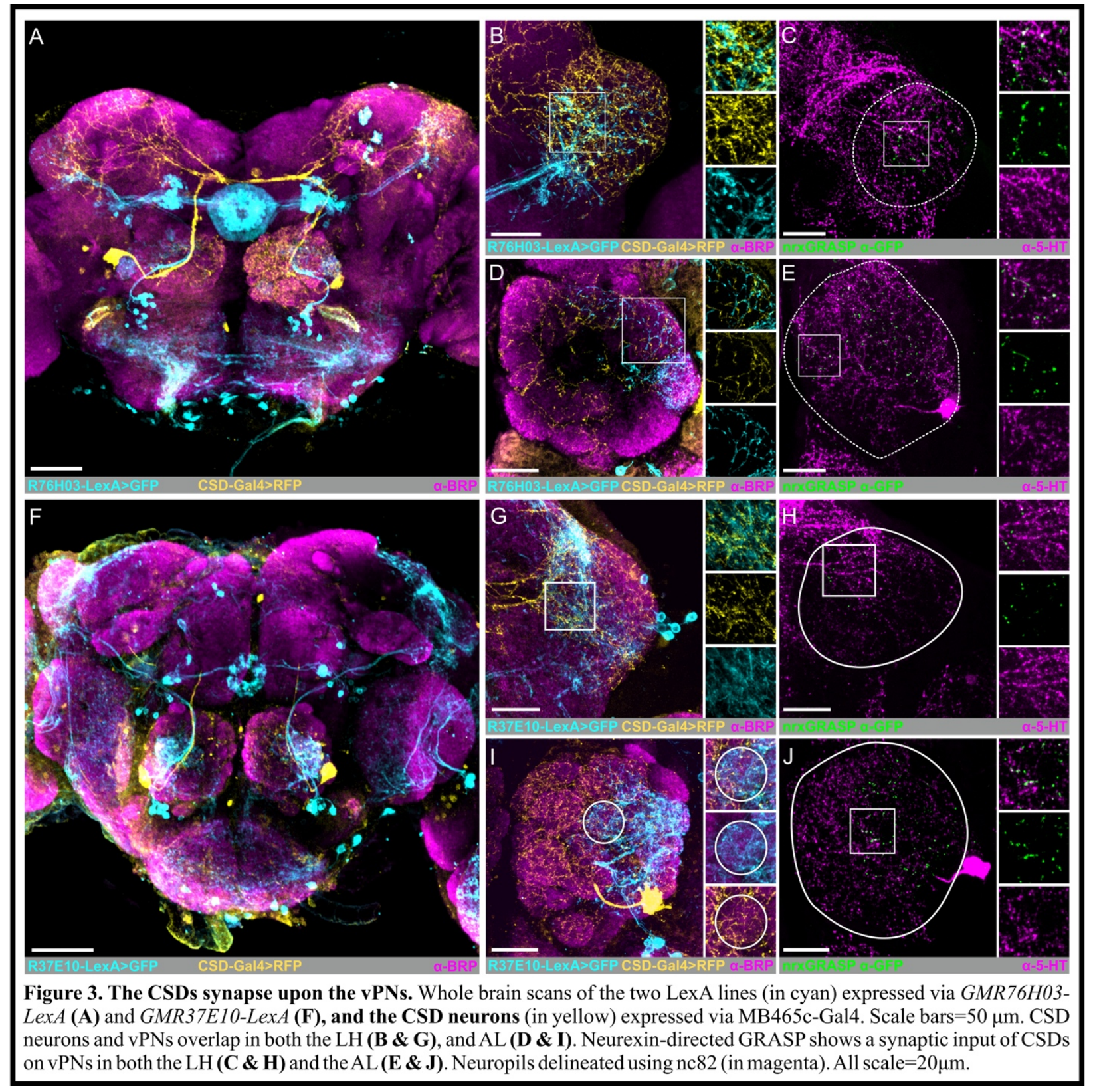




\section{Discussion}

Neuromodulation is crucial for adjusting an organism's behavior based on its physiological state, including hunger (Farhan et al., 2013), time of the day (Linn and Roeloffs, et al., 1986), aggression state (Alekseynko et al., 2010, as well as external cues. Neuromodulatory neurons/nuclei usually project widely throughout the nervous system and can potentially affect multiple circuits simultaneously to adjust their dynamics and activities. For example, in mammals, serotonergic neurons originating in the raphe nuclei extend projections to two synaptic layers in the olfactory system, the olfactory bulb (OB) (Mclean and Shipley, 1987) and the olfactory cortex (Lottem et al., 2016). The neural interconnections between the OB and olfactory cortex are complex, as raphe neurons simultaneously synapse onto different populations of neurons within both regions and can affect them in distinct ways (Brunert et al., 2016; Lottem et al., 2016). Further, both regions receive modulation from other neural circuits (Mclean and Shipley, 1987; Lottem et al., 2016; Brunert et al., 2016). Indeed, despite extensive research, the consequences of neuromodulatory processes across multiple synaptic layers and the nature of their dynamic integration remains largely unexplored. The modulation of vPNs by the CSD neurons is a great model to investigate neuromodulation across synaptic layers for two reasons. First, compared to the Raphe nuclei which are comprised of tens of thousands of neurons, the CSD neurons consist of one pair of identified neurons that are the sole source of synaptic 5-HT in the two olfactory centers, AL and LH (Coates et al., in revision; Zhang \& Gaudry 2016). Secondly, vPNs form exclusive connections between these two centers, and they express all 5-HT receptor types. Thus, due to the relative simplicity and well-characterization of the components of the vPNs/CSD neurons model, in this chapter I use this model to investigate whether it is possible for neurons that span two processing stages to receive synaptic input from a single modulatory neuron. I could determine the olfactory centers in which the CSD neurons provide synaptic input to the vPNs using Neurexin GRASP, which further allowed me to test if the CSDs are presynaptic to the vPNs. My data shows GRASP signal in the AL and LH, suggesting that the CSD neurons synapse on vPNs in both these regions. Collectively, this suggests that vPNs receive dendritic serotonergic modulation in the AL and a weaker axonal serotonergic modulation in the $\mathrm{LH}$. 
The vPN cluster is located ventral to the AL and consists of 51 GABAergic neurons (Wilson and Laurent, 2005; Lai et al., 2008; Strutz et al., 2014). They selectively respond to olfactory information of innately-attractive odors (Strutz et al., 2014; Liang et al., 2013) and silencing vPNs eliminated behavioral attraction in the flies (Strutz, et al., 2014). Studies have shown that vPNs transfer olfactory signals processed in the AL to the LH by extending dendrites to the AL and axons to the LH. (Lai et al., 2008; Tanaka et al., 2012: Parnas et al., 2013). Additionally, Strutz, et al. (2014) reported that most GABAergic vPNs are oligo-glomerular (innervating $\sim 5$ glomeruli each) and send heterogeneous dendritic innervation to AL glomeruli, which is consistent with the projection patterns of the LexA lines I used in this study (Fig. 2 B $_{1}$ $\left.\& \mathrm{D}_{1}\right)$.

The presence of GRASP signal of CSD neurons synapsing onto the dendrites of the vPNs in the AL suggests that the CSD neurons alter the excitability of vPNs via an axo-dendritic mechanism. The question here is, how does endogenous 5-HT released by CSD neurons affect the synaptic integration of ORNs onto vPNs? Endogenous 5-HT has been shown to elicit inhibitory effects on the first olfactory centers in insects and mammals via direct and indirect mechanisms. For instance, a recent study in mice by Huang et al, (2017) showed that 5-HT signaling via the receptor type 5-HT1 elicits direct inhibitory effects on the mitral cells in the OB of mice. Further, endogenous 5-HT released in the olfactory bulb by raphe nuclei neurons was found to inhibit the activities of ORNs indirectly when 5-HT binds to its 5HT-2C receptor type expressed in GABAergic periglomerular cells and causes the release of GABA from them (Petzold, 2009). In Drosophila, optogenetic activation of CSD neurons caused a predominant inhibition in the AL glomeruli (Zhang and Gaudry, 2016). This inhibition is thought to be applied directly to LNs which express the 5-HT receptor types 1A (Sizemore and Dacks, 2016). Based on these observations and on my GRASP data, I suggest an additional direct serotonergic inhibitory mechanism implicated by CSD neurons on vPNs in the AL.

vPNs express all the 5 types of 5-HT receptors (Sizemore and Dacks, 2016) that affect different second messenger pathways. Two receptor types, 5-HT1A and 5-HT1B are GPCRs that are negatively coupled to the second messenger adenylate cyclase (Witz et al., 1990; Saudou et al., 1992; Nichols and Nichols, 2008). Therefore, the suggested inhibition elicited by CSD neurons on vPNs is potentially mediated by one or both these receptors and achieved by reducing 
cAMP concentrations in dendritic terminals of vPNs. However, it should be noted that many vPN neurons also express the other types of 5-HT receptors, 5-HT2A, 2B and 5-HT7 (Sizemore and Dacks, 2016), which are GPCRs coupled with excitatory G subunits (Witz et al., 1990; Saudou et al., 1992; Nichols and Nichols, 2008). Therefore, it is possible that the inhibitory effects elicited by CSD neurons on vPNs are concomitant with excitatory effects. Moreover, the protein trap technique that was used for visualizing the expression of 5-HT receptors in vPNs (Sizemore \& Dacks, 2016) does not provide the specific location at which these receptors are being expressed (AL vs LH) nor it does tell us which vPNs co-express which receptors. Taken together, CSD neurons might be providing compartment specific modulation via differential localization of each 5-HTR expressed by a given vPN.

The LH plays a crucial role in processing innate-odor olfactory information. Olfactory signals about attractive odors are conveyed to the LH by ePNs and vPNs through spatially distinctive pathways (Ito et al., 1997; Stocker et al., 1997; Wang et al., 2014). Specifically, ePNs transfer olfactory information to both MB and LH via mALT and IALT, while vPNs send olfactory information to LH directly via mlALT, bypassing the MB (Lai et al., 2008). Therefore, while MB receive only excitatory input from the ePNs, LH receive parallel excitatory and inhibitory input via ePNs and vPNs, respectively (Jefferis et al., 2001; Lai et al., 2008; Okada et al., 2009: Tanaka et al., 2012; Liang et al.,2013; Wang et al., 2014). In addition, ePN and vPNs, the LH circuitry includes several types of third-order neuron populations. One in particular, the ventrolateral projection neurons (vlPrNs), have been well studied as targets of the vPNs. The Mz699 line labels 86 vlPrNs (Parnas et al., 2013), which send dendrites to the LH and axonal projections to a third order olfactory center called the ventrolateral protocerebrum. vlPrNs play a key role in fly responses to food and pheromone odors (Parnas et al., 2013; Liang et al., 2013). In response to food and pheromone odors, vlPrNs receive excitatory and/or inhibitory inputs from ePNs and vPNs, respectively. The significance of receiving parallel inputs by vlPrNs in the LH and the consequences of changing the balance of inhibition/excitation input received by vlPrNs on odor coding has been explained by two contradicting models that suggest different circuitries (Fig. 4). In the LH, my data shows GRASP signals on the axon terminals of vPNs suggesting a presynaptic axon-axonal modulation by the CSD neurons onto vPNs in this region. The consequences of this modulation on odor integration in the $\mathrm{LH}$, therefore, could be interpreted differently based on each of the two models. 
The first model, presented by Parnas et al, (2013), suggested that vPNs synapse upon the axon terminals of ePNs in the LH (Fig. 4A). They also suggested that the release of GABA from the axon terminals of vPNs causes a decrease in the synaptic strength of ePNs in the LH. The presynaptic inhibition of ePNs by vPNs would serves as a "high-pass" filter mechanism which would enhance the flies' ability to distinguish between closely related odors by suppressing weaker responses to produce a sparser representation. They proposed that this increase would decrease the fly's ability to sense weak olfactory stimuli. Therefore, this inhibition would only be engaged when the fly encounters a strong olfactory stimulus. The GRASP signal I observed in the LH indicates that vPNs receive serotonergic input from CSD neurons. This input could be excitatory or inhibitory based on the receptors expression of vPNs in this compartment. Therefore, CSD neurons could either increase or decrease the quantal content and/or excitability of vPNs, and consequently, increase or decrease the ability of the flies to sense or differentiate between odors. Thus, the CSD neurons could potentially be playing a role in equalizing the balance between two features of the olfactory system, sensitivity and contrast, in the fly. This suggestion could be supported by studies in moths showed that the 5-HT levels fluctuate through the day and are at their highest during the time of day when moths are engaged in odor-guided behavior (Gatellier et al., 2004; Kloppenburg et al., 1999), which suggests a potential role for 5HT in increasing the moths' sensitivity to odors, potentially by shifting the balance off the contrast.

In a second model, Liang et al, (2013) suggested that ePNs provide excitatory synaptic input to vlPrNs in the LH and that vPNs only respond to attractive food odors. They also suggested the presence of a parallel inhibitory mechanism in which vPNs synapse onto vlPrNs, but not the ePNs as is proposed in the Parnas model (Fig. 4B). Since only attractive food odors would elicit activities in vPNs, this inhibition is specific to attractive food odors, but not pheromones. Therefore, the inhibition is absent when the fly encounters a repulsive odor or a pheromone. This selective, parallel inhibitory mechanism, they argued, provides the fly with the ability to segregate biologically important information, such as food and pheromone signals, into different processing channels in the higher olfactory centers. Moreover, it shows a crucial role for vPNs in mediating fly responses to innately attractive odors in the $\mathrm{LH}$, a role that was further supported by Strutz et al, (2014). Based on this model, my data would suggest that 5-HT released by the CSD neurons indirectly modulates the synaptic integration of vlPrNs. That is, the CSDs 
would synapse onto the vPNs, axo-axonically, to alter vPN inhibition of vlPrNs. This could potentially cause two consequences: first, 5-HT can enhance the process of segregating olfactory signals carrying different biological values, i.e. food vs. pheromones, to elicit distinctive behavioral responses depending on the physiological state. Secondly, by altering the synaptic strength of the vPNs, vlPrNs will receive stronger or weaker inhibitory input when the fly encounters attractive odors, which would eventually affect the strength of the fly attraction them (see chapter 3)

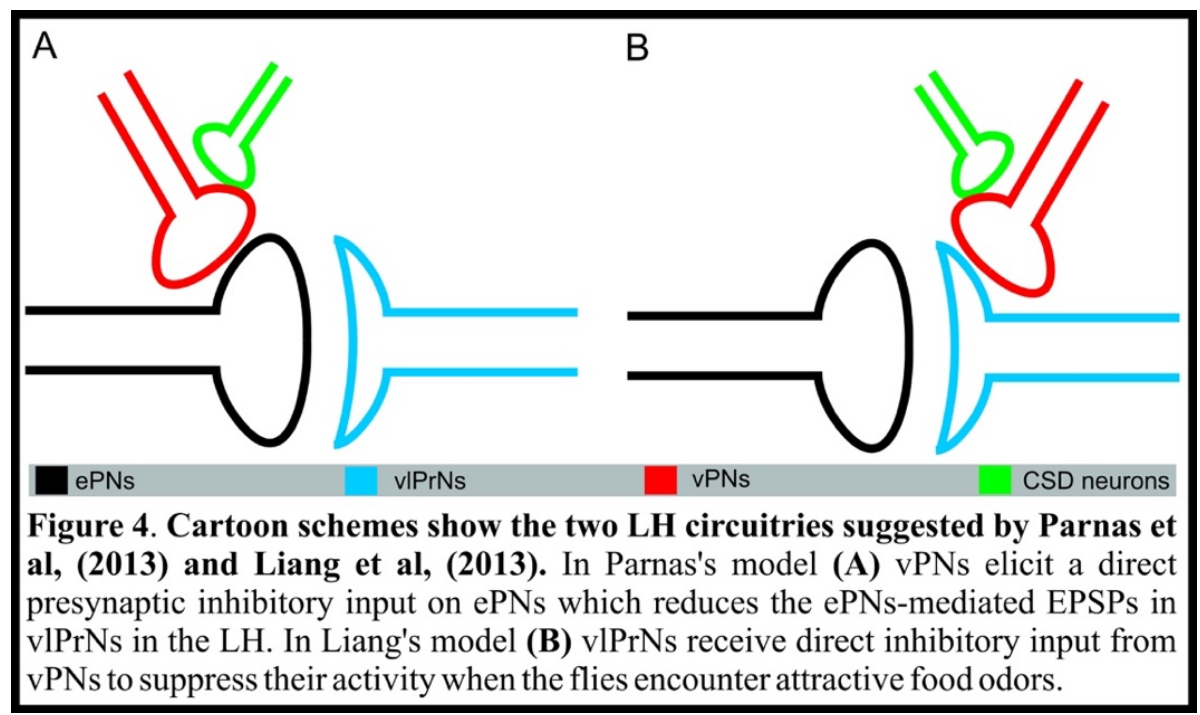

Neuromodulation alters the synaptic or intrinsic properties of a neuron and neuromodulators such as 5-HT could have either an excitatory or inhibitory modulatory effect on the vPNs depending on the receptor type being activated. The 5-HT receptors types encoded the genome of the fly can be categorized as excitatory (5-HT2A, 5-HT2B \& 5-HT7) and inhibitory (5-HT1A \& 5-HT1B) receptor types. 5-HT signaling via these receptors mediates several cellular mechanisms that cause changes in the intrinsic and synaptic properties of target neurons which occur over different time scales. For instance, in Aplysia, tail withdrawal reflexes can be modulated via a serotonergic interneuron that enhances the strength of the synapse between the siphon sensory neuron and the motor neuron responsible for retraction of the gill (Byrne and Kandel, 1996; Klein et al., 1982). The axo-axonic modulation is achieved when 5-HT activates the cAMP second messenger cascade in the siphon sensory neuron (Klein et al., 1982; Byrne and 
Kandel, 1996). cAMP activates PKA, which decreases $\mathrm{K}^{+}$conductance and makes the membrane of the sensory neurons more resistant. The increase in membrane resistance causes an increase in the numbers of action potentials elicited and action potential width, thus, increases $\mathrm{Ca}^{+2}$ influx into the sensory neuron. The increase in $\mathrm{Ca}^{+2}$ influx, in turn, increases the probability of vesicle fusion by mobilizing more vesicles from the reserve pool, thus, enhances the quantal content of the sensory neuron and creates longer and wider EPSPs in the motor neurons (Klein et al., 1982 Byrne and Kandel, 1996). In moths, a 5-HT mediated change in $\mathrm{K}^{+}$conductance was observed in AL neurons in M. sexta (Mercer et al., 1995; Kloppenburg and Hildebrand, 1995). This change, similarly, prolongs the action potential and causes an increase in the influx of $\mathrm{Ca}^{++}$via voltagegated $\mathrm{Ca}^{++}$channels (Mercer et al., 1995), suggesting this serotonergic modulation is likely mediated by the similar cAMP pathway explained in Aplysia.

Another second messenger system that can potentially mediate the 5-HT enhancement of gill withdraw reflex in Aplysia is the membrane associated enzyme PLC. PLC initiates the cleaving of $\mathrm{PIP}_{2}$ to DAG and $\mathrm{IP}_{3}$. DAG activates $\mathrm{PKC}$, which phosphorylates, and thus activates, presynaptic proteins responsible for mobilizing more glutamate-filled vesicles from their reserve pools to become ready to be fused to synaptic membrane. On the other hand, $\mathrm{IP}_{3}$ can also bind to its receptors on the endoplasmic reticulum and cause higher concentrations of $\mathrm{Ca}^{++}$to be released. All previous pathways lead to an increase in the quantal content of the siphon sensory neuron in Aplysia, and potentially in this case, to vPNs. Contrary to 5-HT2 and 5-HT7 receptor types, 5-HT1 type is negatively couple to adenylate cyclase. It reduces the rate at which ATP is converted to cAMP, and thus, elicits inhibitory effects the cell. Based on the type of receptors and the circuitry of the neural networks in which they are expressed, changes in intrinsic and synaptic properties can lead to different behavioral outputs that provide the organism with the ability to adjust its behavior according to its physiological state and environmental stimuli.

Taken together, I demonstrated that CSD neurons synapse onto vPNs in two different synaptic levels, AL and LH, and that CSD neurons provide vPNs with a simultaneous dendritic synaptic modulation in the primary olfactory center, AL, and an axonic synaptic input in the LH. This is an evidence that a one population of neurons spanning two different synaptic layers could simultaneously receive neuromodulatory input from the same source in the two layers. The consequences of the serotonergic modulation of vPNs by the CSD neurons could be interpreted 
differently based on the pattern of connectivity between vPNs, ePNs, and the vlPrNs in the LH. In the model circuitry that was suggested by Parnas and his colleagues (Fig. 4A), the modulation of vPNs by CSD neurons would causes a direct alteration in the neural activity in ePNs, and consequently, in vlPrNs, which affects how the olfactory system process signals related to olfactory system features such as contrast and sensitivity. Converse to this, Liang and Colleagues suggested a different LH circuitry in which vlPrNs receive direct dendritic inhibition from vPNs (Fig. 4B), thus, different consequences of the serotonergic modulation might be resulted. In the next chapter, I will provide a detailed discussion of the behavioral consequences of serotonergic modulation of vPNs output in the LH based on the two previous models. 


\section{Chapter 3: Determine the Contribution of 5-HT1A Receptor Expression by vPNs on Olfaction-mediated Behavior.}

\section{Abstract:}

In an ever-changing environment, an organism must constantly adjust its behavior based on external cues and its internal physiological states. This adaptation is mediated in sensory networks, including the olfactory system, by several neuromodulators such as the monoamine serotonin $(5-\mathrm{HT})$. While the role of serotonergic modulation in insect behavior has been well studied, little is still known about the role of each individual receptor type in modulating innately olfactory responses. Furthermore, the degree to which 5-HT can modulate responses to innately important odors is also not well understood. In Drosophila, one population of olfactory neurons, vPNs, are crucially involved in innate attraction to food odors, and express all 5 types of 5-HT receptors. In this chapter, I use RNA interference to demonstrate the effects of reducing the expression of the 5-HT1A receptor type, in vPNs on flies' responses to innate attractive and repulsive odors. Using two behavioral assays, box trap and T-maze assays, I demonstrate that reducing the expression of 5-HT1A receptors in vPNs increases the flies' attraction to the innate attractive odors apple cider vinegar (ACV) and balsamic vinegar (BV). Further, I show that this reductions in the expression of 5-HT1A does not affect flies' response to aversive odors. Therefore, my data suggests an important modulatory role for endogenous 5-HT elicited by CSD neurons on innate olfactory responses of flies towards attractive food odors mediated via 5HT1A receptor in vPNs.

\section{Introduction:}

Animals' innate responses to environmental stimuli are critical for their survival. Unlike learned responses, innate behavioral responses do not require prior experience. For instance, naive hawkmoths are innately attractive to female pheromones immediately after eclosion. Innate response, however, are subjected to modulation and it is important for these responses to occur in the right context to be most beneficial. For instance, serotonin increases the sensitivity of male moths to female pheromones at certain time of the day which they are searching for mates (Gatellier et al., 2004; Kloppenburg et al., 1999: Linn and Roelofs, 1986). To ensure the efficiency of behavioral responses to innately important stimuli, therefore, animals' sensory 
systems have evolved complicated mechanisms that are regulated by neuromodulators such as 5HT.

5-HT has been found in the primary olfactory centers of both mammals and insects. In mammals, the olfactory bulb $(\mathrm{OB})$ receives dense innervation from the raphe nuclei neurons. These innervations target olfactory glomeruli where they release synaptic 5-HT to modulate their odor-evoked activities. For instance, 5-HT released by the serotonergic axonal varicosities in the OB alters the sensory gain in the olfactory sensory neurons (ORNs) based on the vigilance state of the animal (Petzold et al., 2009). Similarly, in Drosophila, 5-HT released by CSD neurons in the AL modulates olfactory information processing (Dacks et al., 2008; Dacks et al., 2009; Zhang and Gaudry, 2016). The effects of 5-HT on its neuronal targets are determined by distinctive types of receptors. However, the diversity of the individual raphe neurons in their morphology and physiology as well as the diversity of 5-HT receptors in mammals makes the task of studying the functional contribution of each receptor individually to the overall effects of 5-HT on innate behavior extremely challenging. Thus, the use of a simpler system, such as the olfactory system of Drosophila, is ideal. In Drosophila, five 5-HT receptor types are expressed as opposed to 14 subtypes in mammals (Nichols and Nichols, 2008). 5-HTR subtypes are expressed by different classes of AL neurons, these are; 5-HT1A, -1B, -2A, -2B and -7 (Nichols and Nichols, 2008; Blenau and Thamm, 2011). These receptors are coupled to different Gprotein alpha subunits, and thus, they elicit different neuromodulatory effects on target neurons.

In Drosophila, odors are detected by ORNs, which convey olfactory signals to the AL where they are initially processed. Two types of PNs, cholinergic ePNs and inhibitory vPNs, then transfer these signals to higher olfactory centers in the brain, including the mushroom body (MB) and lateral horn (LH). vPNs are exclusively activated by innately attractive odors (Liang, et al., 2013), and are necessary for olfactory attraction (Strutz et al., 2014). Although contradicted to some degree in the precise mechanism, two studies have shared the conclusion that vPNs inhibit third-order LH neurons, the vlPrNs ("ventrolateral Protocerebral neurons"), either directly (Liang et al., 2013) or indirectly (Parnas et al., 2013) to alter the flies' response to innate attractive food odors. The inhibitory input of vPNs to vlPrNs is mediated by release of GABA in the LH (Parnas et al., 2013; Liang et al., 2013), and eliminating the synthesis of GABA in vPNs causes a decrease in flies' attraction to food odors (Strutz et al., 2014). vPNs 
exclusively connect the AL and LH, and collectively express all 5-HT receptor types encoded in the fly genome (Sizemore and Dacks, 2016). In chapter 2, I demonstrated that CSD neurons synapse upon vPNs in both the first- and second-order olfactory centers AL and LH, respectively. This data suggests that $\mathrm{vPNs}$ receive serotonergic modulatory input from the CSD neurons in both centers which likely shapes the flies' response to innately important stimuli. However, it is not known how each of 5-HT receptor types expressed in vPNs individually participates in modulating the innate behavior. In this chapter, my goal was to determine the role of the 5-HT1A receptor expression in vPNs in modulating behavioral responses to innate attractive and aversive odors. I hypothesized that by reducing the inhibitory effect that vPNs receive from CSD neurons, vPNs would potentially elicit more inhibition on vlPrNs, and thus, attraction in flies would be stronger. My data supports my hypothesis and shows that reducing the expression of the inhibitory receptor type 5-HT1A in vPNs increases flies' responses to the naturally attractive odors $\mathrm{ACV}$ and $\mathrm{BV}$, and that rescuing the expression of 5-HT1A in vPNs is sufficient for returning the response to baseline. Further, consistent with recent study showed that vPNs do not respond to aversive odors (Liang et al., 2013; Strutz et al., 2014), my data shows that reducing the 5-HTA1 receptor type did not alter responses to an innately aversive odor, suggesting the absence of a serotonergic modulatory mechanism on aversion in flies.

\section{Materials \& Methods}

Fly stocks: Flies were maintained on a Nutri-Fly "German Food” Sick Fly Formulation at $50 \%$ humidity and a $25^{\circ} \mathrm{C}$ on a $12: 12$ dark-light cycle. The following fly lines were used: Mz699-Gal4 (provided by Dr. Tzumin Lee), UAS-5HT1a-RNAi (BDSC \#33885), and UAS5HT1a (BDSC \#27630). Mz699-Gal4 and UAS-5HT1a-RNAi or were used as parental lines in all behavioral experiments.

Rescue Stable line: The stable line UAS-5HT1a-RNAi;UAS-5HT1a was established by crossing female virgins from each of UAS-5HT1a-RNAi and UAS-5HT1a lines individually with the balancer line 2475. F1 progenies from both crosses with specific traits (to ensure that no recombination processes across the genome of the flies had occurred) were then collected and self-crossed as described in Chapter 2. Finally, F2 progenies from both crosses were collected and crossed with each other to generate the final stable line. The rescue line was maintained on the same fly food and conditions mentioned above. 
Table 2. Drosophila genotypes used in chapter 3.

\begin{tabular}{|c|c|}
\hline Figure \# & Genotypes (Transgenes with Bloomington number) \\
\hline Figure $5 \mathrm{~B} \& 5 \mathrm{E}$ & W1118 wild type (BDSC \#6326) \\
\hline Figure $5 \mathrm{C}, 5 \mathrm{~F}, 5 \mathrm{H}-\mathrm{I}$ & $\begin{array}{l}\text { Parental lines: } \\
\text { - Mz699-Gal4 (provided by Dr. Tzumin Lee) } \\
\text { - UAS-5HT1A-RNAi (BDSC \#33885) } \\
\text { F1 flies: } \\
\text { - UAS-5HT1A-RNAi;Mz699-Gal4 }\end{array}$ \\
\hline Figure $5 \mathrm{G}$ & $\begin{array}{l}\text { Parental lines: } \\
\text { - Mz699-Gal4 (provided by Dr. Tzumin Lee) } \\
\text { - UAS-5HT1A-RNAi (BDSC \#33885);UAS-5HT1A (BDSC \#27630) } \\
\text { F1 flies: } \\
\text { - UAS-5HT1A-RNAi;UAS-5HT1A/Mz699-Gal4 }\end{array}$ \\
\hline
\end{tabular}

Box Trap Assay: A 1000 microliters pipette tips box was used to construct the trap. The box has a tray that was completely taped closed except of two holes in it. The two holes were (30) $\mathrm{mm}$ and (50) mm away from the edges and from each other, respectively. Two cones were constructed by cutting $25 \mathrm{~mL} 15 \mathrm{~mL}$ tubes (Thermo Scientific, Cat. \#362695) at the mark of 2 $\mathrm{mL}$ and cutting their closed ends at the tip. The wide end of the cones was $\sim 15 \mathrm{~mm}$ while the small end was $\sim 4 \mathrm{~mm}$. The wide ends of the cones were then secured to the holes from the inner side of the tray using dental wax (Darby Dental Supply, LLC, Cat. \#952-2422). The same wax was also used to secure two scintillation vials to the cones so that the small ends point towards inside of the vials. The boxes were labeled with small pieces of tapes indicating the contents of the vials (i.e. water vs. ACV) as well as the concentrations of the odor solutions. A 200 microliter of water (control) or odor was pipetted into the vials. A range of six different concentrations were tested; 1 in 1000, 1 in 200, 1 in 100, 1 in 10,1 in 20 and pure.

All the experiments were conducted during the active morning period (9-11am). The flies were starved in tubes containing wet Kim wipes for 24 hours before testing them. Tubes containing starved flies were set in ice for 3-7 minutes to reduce flies motor activities before moving the files out to the white trays of the boxes. The boxes were then closed and put in an 
incubator in a 32C temperature, 50-60\% humidity, and in dark. Flies were given 120 minutes to choose between walking into one of the scintillation vials before they were removed to the freezer. The number of the flies on the white top, in both vials were counted and the performance indices calculated according to the following equation:

(\# of flies in odor vial - \# of flies in control vial)/(\# of flies participate in the experiment)

All statistical calculations were done using Graph Pad Prism v.6.01 (Graphpad Software Inc.). To determine the normality of the data collected, A D'Agostino-Pearson omnibus normality test was done. All data in this study were found to be non-normally distributed and thus a Kruskal-Wallis (one-way ANOVAs) test was used to determine the presence of significant responses between lines at each odor concentration.

Box Trap Cleaning: After counting the flies, traps were disassembled and the scintillation vials were soaked in hot water with soap for 10-15 minutes. A small brush was then used to scrub vials thoroughly, before rinsing the vials extensively. The vials, boxes, cones, and taped trays were then sprayed with $70 \%$ Ethanol. The boxes and the taped trays were also wiped with Kim wipes to ensure a complete distribution of alcohol on all surfaces, and all components were placed in the oven at $48 \mathrm{C}$ overnight to dry.

T-maze assay: The T-maze was constructed by drilling holes $(\sim 4 \mathrm{~mm}$ in diameter $)$ in the caps of two bacteria culture tubes $(12 \times 75 \mathrm{~mm}$ Polystyrene Tubes with Dual Positions Polyethylene Caps, USA Scientific, Cat. \#8576) tubes. The two caps were then secured to the horizontal arms of a Tjunction. The $\mathrm{T}$ junction contained a small funnel was constructed by cutting a fine transfer pipette (Samco Scientific, Cat. \#232-11) and used to secure the vertical introductory tube to the T-junction. Two 5 × $5 \mathrm{~mm}$ pieces of filter papers were cut. Each piece was put in one of the horizontal arms and 30 micro liters of either tested odor or solvent were pipetted on them. A range of odor concentrations spanning the spectrum of odor-attracted responses, with the lowest concertation elicits no attractive responses and the highest concentration evokes a maximum response, was first established in wild type (W1118) flies (Fig. 5E). Same dilutions series was then tested on experimental flies. Every 10 days, 90-120 female virgins from the UAS-5HT1aRNAi were collected and crossed to 60-90 male flies from the Mz699-Gal4 line in three 150ml plastic bottles ( $\sim 35$ female virgins $\& \sim 20$ males in each bottle) containing same standard fly food formula. The flies were then pushed to three new bottles every 3 day for a maximum of three 
times before being tossed and a new cross between the parental lines was done. F1 flies were maintained at the at $50 \%$ humidity and a $25^{\circ} \mathrm{C}$ on a $12: 12$ dark-light cycle.

The two parental lines and their F1 progeny were all collected (Table 3) and tested simultaneously, but in different assays. The behavioral assay was done by starving mated female flies first for 18-20 hours in groups of 25 in fly tubes (VWR-Drosophila Vial, Cat. \#3815) containing wet Kim wipes (Distilled water was used). All preparation for the T-maze assays were done under red-light conditions to prevent light from influencing the flies' choices. At the beginning of the assay, the files were cold-anaesthetized by putting starvation tubes on ice for 37 minutes. Flies were then placed in the bottom of the introductory tube of the T-maze using a small funnel. The T-mazes were then assembled and placed vertically in microcentrifuge tube racks and put in an incubator in dark at $32^{\circ} \mathrm{C}$ and $60-70 \%$ humidity. The orientation of the $\mathrm{T}$ maze arms containing the odor versus solvent were alternated spatially to eliminate any directional bias. The flies were giving 45 minutes to walk up and choose between the two arms. The number of flies participated in the experiment, determined by counting the number of flies entered both arms, were noted to ensure the absence of any motor deficits. Once an arm was chosen, the flies were trapped and not able to walk back into the T-junction because of a taper in the T-junction arms. Upon completion of the assay, the T-mazes were taken out of the incubator and placed in the freezer for approximately 90 minutes. the number of flies in each arm were then counted. Performance indices were finally calculated by following the equation:

(\# of flies in odor arm - \# of flies in control arm)/(\# of flies participating).

Microsoft ${ }^{\circledR}$ Excel was used for initial entry of data. However, all statistical analyses were done using Graph Pad Prism v.6.01 (GraphPad Software Inc.). To determine the normality of the data collected, A D'Agostino-Pearson omnibus normality test was done. All data in this study were found to be non-normally distributed and thus a Kruskal-Wallis (one-way ANOVAs) test was used to determine the presence of significant responses between lines at each odor concentration. 
Table 3. T-maze working schedule

\begin{tabular}{|c|c|c|c|c|c|}
\hline Day 1 & Day 2 & $\begin{array}{c}\text { Day } \\
\mathbf{3}\end{array}$ & $\begin{array}{c}\text { Day } \\
4\end{array}$ & Day 5 & Day 6 \\
\hline $\begin{array}{l}\text {-Collect } 1 \text { day } \\
\text { old female } \\
\text { flies (A) }\end{array}$ & $\begin{array}{l}\text {-Collect } 1 \text { day } \\
\text { old female } \\
\text { flies (B) }\end{array}$ & & & $\begin{array}{l}\text {-Starve flies at } 2 \mathrm{pm} \text { (if } \\
\text { assay is at } 10 \mathrm{am} \text { ) } \\
-\mathrm{A}=5 \text { days old }-\mathrm{B}=4 \text { days } \\
\text { old } \\
\text {-Make sure all T-maze } \\
\text { components are clean, dry, } \\
\text { and ready to go }\end{array}$ & $\begin{array}{l}\text {-T-maze assay } \\
@ 10 \text { am } \\
\text {-(preparations } \\
\text { start @ 9:00 } \\
\text { am) } \\
-\mathrm{A}=6 \text { days old, } \\
-\mathrm{B}=5 \text { days old }\end{array}$ \\
\hline
\end{tabular}

T-maze Cleaning with Alconox: On day 1: 15g (pre-measured) Alconox were dissolved into 1500-1800 mL of hot tap water in the clean plastic pitcher. T-maze components were then added and vigorously swish around for at least 30s, ensuring all tubes have soapy water in them and then let soaked for overnight. On day 2 , a small brush was used to scrub the tubes very well. The soapy water was then drained using white strainer and components were rinsed under warm-hot tap water. The rinsing step were done several times (at least three times). After that, tap water was added to pitcher and components were soaked for 10-15 minutes before water was then drained and components were rinsed again thoroughly. Finally, all components were sprayed with $70 \% \mathrm{EtOH}$ before being rinsed thoroughly with distilled, de-ionized water, drained, and placed in oven at $48.5^{\circ} \mathrm{C}$ overnight

\section{Results}

I previously demonstrated that the CSD neurons synapse onto the vPNs (Chapter 2) and a study from our lab demonstrated that the vPNs express several 5-HT receptors (Sizemore and Dacks, 2016). However, the contributions of these individual receptors expressed in vPNs to olfactory guided behavior is unknown. Therefore, I sought to determine the contribution of 5HT1A receptor expression by vPNs on flies' behavior towards innately attractive and repulsive odors. A pilot behavioral study from our lab showed that reducing the expression of 5-HT1A receptor in vPNs increases flies' attraction to high concentrations of the innately attractive odor, 
apple cider vinegar (ACV) in the box trap assay. This suggests that 5-HT from the CSDs may modulate innate olfactory guided behavior via the vPNs. Therefore, I sought to expand on this initial pilot study to determine the behavioral consequences of 5-HT1A knockdown in the vPNs.

Mz699-Gal4 has been used in several studies to determine the contribution of the vPNs to olfactory coding in the LH and innate behavior (Parnas et al., 2013; Strutz, et al., 2014). Mz699 line drives Gal4 expression in both vPNs and vlPrNs. However, unlike vPNs, vlPrNs do not generate the neurotransmitter GABA (Parnas, et al., 2013) neither do they express any of the 5HT receptor types (Sizemore, unpublished). Therefore, I could safely use the Mz699-Gal4 line to drive the expression of the UAS-5-HT1A-RNA interference specifically in vPNs to investigate the consequences of reducing the expression of the 5-HT1A receptor type on the behavioral responses of the flies. Several studies have shown this technique is effective to knock down the 5HT-type receptors in flies (Shimada-Niwa and Niwa, 2014). Furthermore, data from our lab has shown using qPCR that the UAS-5-HT1A-RNAi caused a 65\% knockdown of 5-HT1A mRNA copy number when expressed in a pan-neuronal driver line (ELAV-Gal4) (unpublished data).

To test the innate behavioral response of flies to odors, I used two behavioral assays; boxtrap (Knaden, et al., 2013) (Fig. 5A) and T-maze assays (Stensmyr et al., 2012) (Fig. 5D). Both are standard assays that I used in parallel to control for any assay-specific effects. I began by optimizing box-trap assay protocol developed previously in our lab, using wildtype flies (W1118). I tested their responses to several odor concentrations of the innate attractive odor ACV over a range which elicited little to no response (vehicle) to a maximal response. I found a constant, gradual increase in wild type flies' responses concomitant with the increase in ACV concentrations using a range from (water), 1:1000, 1:200, 1:100, 1:20, and pure (Fig. 5B). This data was used as a baseline to compare the behavioral responses of experimental lines. Next I tested the behavioral responses of the experimental lines, Mz699-Gal4 and UAS-5-HT1A-RNAi, as well as their F1 (Mz699-Gal4>UAS-5-HT1A-RNAi) progeny to the same concentrations of $\mathrm{ACV}$ in the box-trap assay. I found that responses of F1 flies were not significantly different from control flies at any of the tested concentrations (1:1000, 1:200, 1:100, 1:20 and pure) of $\operatorname{ACV}$ (Fig. 2C). 


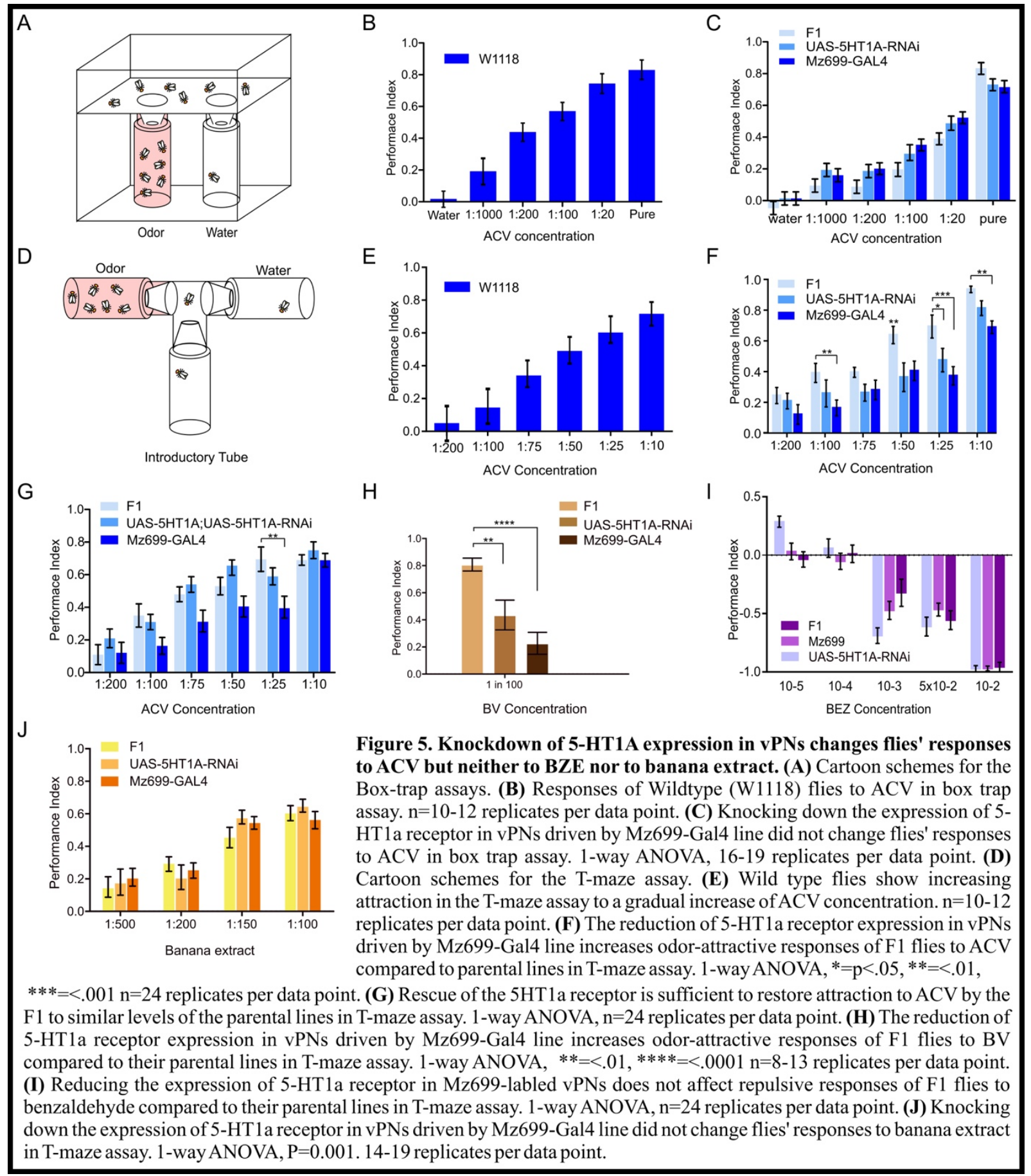


Next, I used the T-maze assay to investigate flies' response to ACV. In T-maze assay, the air space in which the odor can diffuses in is much smaller than in box-trap assay, which makes the T-maze assay a more sensitive assay to study flies' responses to odors. As with the box trap assay, I first tested the responses of wild type flies (W1118) to ACV, choosing 7 concentrations, ranging from maximal behavioral response to no preference over vehicle. The concentrations were: (1:200, 1:100, 1:75, 1:50, 1:25 and 1:10 and pure). The pure concentration was discarded, because it elicited a lower behavioral response than the concentration 1:50, 1:25 and 1:10 which suggests that ACV became aversive potentially due to the activation of additional glomeruli including DM5 (Semmelhack and Wang, 2009) (Fig. 5E). Next, I tested the two parental lines Mz699-Gal4 and UAS-5-HT1A-RNAi as well as their F1 (Mz699-Gal4>UAS-5-HT1A-RNAi) progeny and found that, at the three lowest concentrations, 1:200, 1:100, and 1:75 there was no significant difference between responses of F1 flies and the parental lines. However, F1 flies showed significantly more attractive responses to higher ACV concentrations than both parental lines at the concentrations 1:50 and 1:25, and only higher than the Mz699 at the concentration $1: 10$ (Fig. 5F).

Next, to establish the sufficiency of the 5-HT1A receptor to elicit the observed changes in attractive behavior, I did a rescue experiment. Here, I tested flies bearing the genotype $U A S$ 5HT1A-RNAi;UAS-5HT1A, as well as Mz699-Gal4 and their F1 progeny in T-maze assay. I found no significant difference in responses between the two parental lines and the F1 progeny at any of the ACV concentrations tested (Fig. 5G). Thus, by conducting the rescue experiment, olfactory performance of flies went back to that similar to control (wild type) flies, which demonstrate that the expression of the 5-HT1A receptor in vPNs is sufficient for maintaining normal attractive behavior to the innate attractive odor ACV. Finally, I tested flies' responses to $\mathrm{BV}$, an odor that has the same biological value of ACV, at one concentration only ( 1 in 100). Similar to ACV, I found a significant different between F1 flies attractive responses to the BV compared to their parental lines (Fig. 5H)

Liang et al, (2013) and Strutz et al, (2014) both showed that vPNs respond to innately attractive, but not aversive, odors. Therefore, serotonergic modulation of vPNs by CSD neurons should not have any impact on flies' behavioral responses to innately aversive odors. To test this, 
I wanted to investigate the behavioral consequences of reducing the expression of 5-HT1A receptor in vPNs on flies' responses to the well-known, innate fly repellent benzaldehyde (BEZ) (Stensmyr et al., 2012). BEZ has been successfully used to test flies' responses to aversive odors in T-maze assays (Strutz et al., 2014) and box-trap assay (Knaden et al., 2012). Therefore, I used the T-maze assay to test the behavioral responses of the parental lines, UAS-5-HTIA-RNAi and Mz699-Gal4, as well as their F1 progeny and found no significant differences in the behavioral responses of the two parental lines and the F1 progeny at any of the BEZ concentrations tested (Fig. 5I). This data is consistent with previous studies in which repulsive odors did not elicit neural activities in vPNs, and that vPNs response primarily to attractive odors (Liang et al., 2013; Strutz et al., 2014).

Next, I sought to determine whether reducing the expression of 5-HT1A in vPNs would alter flies' responses to other attractive odors. Therefore, I tested the responses of wild type flies (W1118) to an increase concentration of banana extract dilutions. Unlike ACV, when I tested the behavioral responses of the two parental lines and their F1 progeny there was no significant differences between any of them at any of the tested concentrations (Fig. 5J).

Thus, based on the data I provide above I demonstrate that, reducing the expression of the 5-HT1A receptor type increases attraction but has no effect on the flies responses to repulsive odor. Additionally, I show that the increase in flies' attraction was only to ACV, but not banana odor, which suggests a modulatory role for 5-HT1A expressed in vPNs to odor with specific ecological meaning rather than a generalized modulation.

\section{Discussion}

Innate behavioral responses provide the animal at early stages of its life with the ability to survive by responding to vital stimuli without needing previous exposure to them. For instance, naive male moths are attracted to sex pheromones released by females immediately after they hatch (Saveer et al., 2012). These responses are so critical to the animals' survival, that they have evolved overtime and gained several fine-tuning mechanisms to be more effective. Mainly, these responses became plastic and vulnerable to modulation to best fit the organism's ecological and physiological needs. For instance, in the moth, Agrotis ipsilon, a blend of female sex pheromone and host plants' volatiles strongly attracts males for mating. However, after copulation, the sensitivity of the newly mated male moths to the female pheromones decreases (Barrozo et al., 
2010). Furthermore, these pheromones, although still attractive to males, produce a transient inhibition in the attraction to plants' volatiles which delay re-mating and allow reproductive glands to be refilled again for a later copulation (Barrozo et al., 2010). It has been demonstrated that neuromodulators can provide sensory systems with plasticity to adjust the animals' responses to innately important stimuli. For instance, 5-HT is widely distributed across mammals' nervous systems and plays an important role in regulating the dynamics of sensory networks such as the olfactory system (Lottem et al., 2016; Huang, et al., 2017). While the modulatory effects of 5-HT in mammals are mediated by 14 distinctive receptor subtypes (Nichols and Nichols, 2008), in the olfactory system of Drosophila, only five homologous 5-HT receptor types have been found. These receptors receive their endogenous serotonergic input from two serotonergic neurons, the CSD neurons, which contralaterally project throughout the brain and are the sole sources of 5-HT to several olfactory regions, including the AL and LH (Dacks et al., 2006; Coates et al., in revision). The overall modulatory influence of 5-HT on innate behavioral responses in insects has long been investigated. For instance, exogenous 5-HT has been shown to increase the sensitivity of male Bombyx mori (Gatellier et al., 2004), and Manduca sexta (Kloppenburg and Mercer, 2007) moths to the female sex pheromones. However, there are several questions remain to be explored; First, what is the importance of possessing broad serotonin receptor diversity in a sensory network? Second, what is the contribution of each receptor expressed by each neuron type to the overall effect elicited on innate behavior? Finally, to what degree 5-HT can change an innate behavioral response? In this chapter, I use the olfactory system of Drosophila to explore these questions by investigating the extent to which a single 5-HT receptor expressed by a population of neurons that respond to innately important stimulus can affect the behavioral responses to these stimuli. Specifically, I determine how the 5HT1A receptor, modulates a specific group of inhibitory neurons, vPNs, in the olfactory system to alter behavioral responses to odors that have innate attractive or aversive importance. My data shows that reducing the expression of 5-HT1A receptor increases the attraction of flies to the innately attractive odors, ACV and BV. I also show that flies' responses to innate aversive odors, which don't activate vPNs, are not affected by reducing the expression of 5-HT1A receptor in vPNs. This also suggests that a single 5-HT receptor type can selectively modulate olfactory responses with different innate biological meanings (attraction vs. aversion). 
There are two second-order centers, MB and LH, in the olfactory system of Drosophila. The MB receives only excitatory signals from two different clusters of ePNs located anterodorsally and laterally to the AL (Jefferis et al., 2001, 2007). Chemical ablation of the MB by feeding hydroxyurea to newly hatched larvae caused the loss of adult flies' ability to perform in classic conditioning paradigms (de Belle and Heisenberg, 1994), which established its role in associative learning and memory. On the other hand, LH receive parallel excitatory and inhibitory signals via ePNs and vPNs, respectively (Stocker et al., 2007; Liang et al., 2013), and is known for its role in directing odor-driven innate behavior (de Belle and Heisenberg, 1994; Jefferis et al., 2007; Strutz et al., 2014). In addition to the axon terminals of ePNs and vPNs, the LH circuitry includes several other local and third-order neurons. One LH neuron population, vlPrNs, has been well-studied (Parnas et al., 2013; Liang et al., 2013; Strutz et al., 2014). In the vlPrNs cluster, there are 86 Mz699-positives, non-GABAergic neurons (Parnas et al., 2013) that send postsynaptic terminals to the LH and axon terminals to the ventrolateral protocerebrum. While vlPrNs integrate cholinergic excitatory input from ePNs about attractive, aversive, and pheromone odors, they selectively integrate inhibitory input from GABAergic vPNs about attractive odors (Liang et al., 2013; Strutz et al., 2014). Thus, when the fly encounters attractive food odors, vPNs suppress vlPrNs responses (Liang et al., 2013), to elicit attraction (Strutz et al., 2014).

In chapter 2 , I demonstrated that vPNs potentially receive synaptic input from the CSD neurons at two processing stages in the olfactory system (see chapter 2). Further, my behavioral data suggests that 5-HT signaling via the 5-HT1A receptor type affects processing of innately attractive stimuli. However, since the exact cellular compartment (axons vs. dendrites) in which vPNs receive the 5-HT1A-mediated input from CSD neurons is not known, there are three possibilities; First it is possible that vPNs express 5-HT1A only in the AL. A second possibility is that vPNs express 5-HT1A only in the LH. Third, it is possible that vPNs express 5-HT1A receptors in both the AL and LH (see chapter 2). My GRASP data shows a stronger signal in the AL than in the LH (see chapter 2). Therefore, of these 3 possibilities, it is likely that CSD neurons elicit a direct, axo-dendritic 5-HT-mediated inhibitory input on vPNs in the first olfactory center, AL, where 5-HT1A receptors are potentially expressed. This suggestion is consistent with a recent study in mice showed that 5-HT signaling via the receptor type 5-HT1 elicits direct inhibitory effects on the mitral cells in the OB (Huang et al., 2017). 
Regardless of the location in which vPNs receive the 5-HT-mediated inhibitory input, decreasing the expression of 5-HT1A in vPNs would potentially decrease the inhibition elicited by CSD neurons on them. Consequently, vPNs odor-evoked activity, and their GABAergic output in the LH, would increase. The behavioral consequences of serotonergic modulation of the inhibitory output of vPNs in the LH (i.e. the increase in flies' attraction to ACV that my data shows) as well as the circuitry underlying this increase can be discussed based on two contradicting models set by recent studies.

The first model was set by Parnas et al., (2013) (Fig. 4A), and suggested a presynaptic gain control mechanism in which release of GABA from vPNs decreases the synaptic output of ePNs onto vlPrNs in the manner of a "high-pass filter", so that GABA release from vPNs will only change ePNs synaptic output when ePNs are activated at high levels. This gain control mechanism causes the flies to be more capable of discriminating between odors, especially those of close biological relevance. This increase in contrast however, comes at a cost, which is reducing flies' sensitivity to odors. In this model, serotonergic modulation of vPNs by CSD neurons could potentially be playing a role in balancing the two olfactory system features, contrast and sensitivity. Specifically, one would expect that by increasing the vPNs' odorevoked activities through knocking down their 5-HT1A receptors, the contrast would be increased and the flies would become less sensitive to an innate odor, such as ACV. That is, in behavioral assays data, such decrease in sensitivity, if existed, would be displayed as a decrease in flies' attraction to ACV at low concentrations. My data obtained using T-maze assay does not support this model since it did not show such decrease in flies' responses. Quite the opposite, my data showed a trend in which F1 flies' responses were generally higher than the two parental lines at concentrations below 1 in 50 (significantly higher than the response of one of the parental lines, Mz699-Gal4, at the concentration 1 in 100) (Fig. 5F). However, since my behavioral assays do not test for discrimination, whether my data perfectly fits or refutes this model is not determined and further research should be done (see chapter 4).

Gain control modulation could be playing different roles other than the one suggested in Parnas' model. For instance, in the AL of Drosophila, Root et al., (2008) explained a gain control mechanism on ORNs' terminals mediated by the release of GABA from local interneurons. This mechanism modulates the synaptic transmission of ORNs to PNs and was 
found necessary for the flies' ability to locate odor objects. Further, they explained that ORN-toePN gain modulation in the AL is selectively applied to pheromone-sensing ORNs, as well as ORNs that are potentially important for the localization of attractive odor sources. Therefore, the suggested presynaptic gain modulation of ePNs in the LH could be functioning as a second safety mechanism to insure the "sharpness" of the internal neural image of the innately attractive stimuli to further enhance the fly's ability to locate their sources. Interestingly, ePNs responding to attractive odors and vPNs both extend innervations to the same glomeruli in the AL, and send axons that terminate in the same regions in the LH (Strutz et al., 2014). Therefore, another potential role of the presynaptic gain modulation of ePNs in LH could be working as a second safety mechanism, in addition to the one in the AL, to ensure that ePN responses to attractive odors won't reach saturation, which would happen and erroneously elicit aversion in flies, when additional glomeruli in the AL are activated by high concentrations of same odor (Semmelhack and Wang, 2009). These suggestions fit well with my data in which I show that reducing 5HT1A expression in vPNs, which potentially causes an increase in gain control effects of vPNs on ePNs, increases flies' attraction to ACV. In other words, the serotonergic modulation of vPNs by CSD neurons could be important for modulating the gain control in the innate odor processing center, LH, to sharpen innate odors internal images in it and/or prevent the saturation of innate attractive odors olfactory channels, and thus, fine-tuning the appropriate behavioral response.

The second model was set by Liang et al, (2013) (Fig. 4B), and further supported by (Strutz et al., 2014). In this model, it was suggested that vlPrNs receive direct inhibitory input from vPNs in the LH. Further, they suggested that this inhibition is selective to attractive food odor only. Therefore, pheromone and/or repulsive food would not evoke the activation of vPNs. The inhibition elicited by vPNs onto vlPrNs provides a mechanism for controlling the strength of flies' attraction to innately attractive odors (Liang et al., 2013; Strutz et al., 2014). This model fits well with my data which shows an increase in flies' attractive responses to ACV and BV after reducing vPNs expression of 5-HT1A. Reducing 5-HT1A expression in vPNs potentially removes the inhibitory action of the CSD neurons, thus, increasing their GABA release in the LH, which in turn, causes a greater inhibition of vlPrNs. My data is also consistent with a study was done by Strutz et al., in which they used the same model set by Liang et al., 2013 and showed that disrupting GABA synthesis in vPNs, and thus removing inhibitory effects elicited 
by vPNs on vlPrNs in the LH, eliminated attraction to innate attractive odors. Behavioral studies in the silk moths, Bombyx mori, have shown that 5-HT modulates the olfactory sensitivity of males so that they become more sensitive to females' sex-pheromones (Gatellier et al., 2004). Furthermore, several studies showed that the level of the 5-HT in the AL of Lepidoptera fluctuates throughout the day, and reaches its highest at the time when the moths are actively searching for a host flower or a mate (Gatellier et al., 2004; Kloppenburg et al., 1999). Consistent with these studies, therefore, my data suggests a modulatory role of 5-HT potentially used for modulating the flies' attraction to innate attractive odors at a certain time when a critical odor-guided behavior, such as foraging, is required.

My data also shows that reducing vPNs expression of 5-HT1A does not affect flies' responses to BEZ (Fig. 5I). Why is 5-HT1A modulation of aversion absent in the flies? vPNs do not respond to aversive food odors (Liang et al., 2013). Therefore, it is conceivable that modulating the vPNs activities would not affect flies' responses to aversive odors. One potential explanation for the absence of the modulation of behavioral aversion is that repulsive odors typically imply that there is "danger" and if the insects don't respond promptly, the consequences could be harmful. That is, detrimental consequences (death, inability to mate) could accompany a lack or latency in a quick behavioral response, and thus, innate responses to repulsive odors should be more rigid (i.e. lack plasticity). On the other side of the spectrum, innately attractive odors likely are experienced under different contexts and require greater flexibility or sensitivity to different odors. For instance, unmated cotton leafworm moths innately prefer lilac flowers. However, this innate attraction is shifted to green leaf odors of cotton after the females are mated (Saveer et al., 2012). Using calcium imaging, the group demonstrated that this behavioral switch was due to downregulation of neuronal activity within specific glomeruli that are distinctly responsive to the odors of the lilac flower. This downregulation, they argued, could be attributed to gain control mechanism elicited by LNs on PNs in the AL and mediated by biogenic amines such as 5-HT (Saveer et al., 2012).

Taken together, in this chapter I demonstrated that reducing the expression of the receptor type 5-HT1A in vPNs increases flies' responses to ACV and BV but not to BEZ. Thus, my data suggests a 5-HT1A-mediated modulatory role for endogenous 5-HT on attractive responses to odors with specific biological values to potentially create state-dependent behavioral responses. 
This study, therefore, provide a step forward towards a better understanding of the molecular basis of serotonergic neuromodulation and its contribution to the perception of innate odors in the olfactory system of Drosophila. 


\section{Chapter 4: Future directions}

In this study, I used two different behavioral assays in conjunction with anatomical methods and genetic techniques to investigate brain centers in which a population of neurons critical for flies' responses to innately attractive odors receive serotonergic modulation. Further, I determined the degree to which the synaptic 5-HT modulation can alter these innate responses as well as the behavioral consequences of knocking down one 5-HT receptor type 5-HT1A.

In chapter 2, I demonstrated that, in Drosophila, the CSD neurons synapse upon a population of GABAergic neurons, vPNs, in two olfactory centers, AL and LH (Fig. 3). This suggests that vPNs receive a direct synaptic 5-HT modulation from CSD neurons in both sites simultaneously. However, since vPNs express all 5-HT receptor types (Sizemore and Dacks, 2016) and the location at which these receptors are expressed (AL or LH) was not determined in this thesis, it is unknown which 5-HT receptor types are being expressed at these synapses. Therefore, we do not know the sum effect of 5-HT signaling via its receptor types on vPNs elicited in the AL and/or LH. So far, I am not aware of any genetic tools or antibodies that could be used to label any of the 5-HT receptor types. However, using antibodies that specifically label each receptor types in addition to GRASP technique to determine the type of neurons synapsing on each other in the future not only would be beneficial to further dissect the molecular mechanism of serotonergic modulation of vPNs and the type of modulation (excitatory vs. inhibitory) that occurs at each of the two processing stages in the brain, but also for providing a better understanding of the circuitry of ePNs, vPNs, and vlPrNs in the LH. For instance, Strutz et al, (2014) used $\mathrm{Ca}^{2+}$ imaging to show that the LH comprises of 3 different compartments, LHAM, LH-PM, and LH-AL, that integrate olfactory information related to odor attraction, odor intensity, and negative valence \& odor intensity, respectively. The ability of using targeted antibodies to label each of the 5-HT receptor types individually would allow us to reveal the LH region at which CDS neurons synapse on vPNs and thus could be useful for better understanding the integration of innate odor information in the LH. For instance, although not quantified, the GRASP signals I observed in the LH are localized in LH-PM and LH-AM compartments, suggesting that CSD neurons synapse upon vPNs in these two regions. This observation supports a potential role for endogenous 5-HT via its receptors types in regulating the feature of attraction 
and sensitivity, in the LH. However, the type of receptors expressed at the synapses in both regions is unkonwn. Using a 5-HT1A-specific antibody, it could be revealed whether 5-HT1A is expressed at the synapses in the LH-PM region, which would support my data which showed the implication of a 5-HT1A-mediate inhibitory role elicited by endogenous 5-HT on vPNs to mediate attraction.

In Drosophila, it has been demonstrated that exogenous 5-HT increases activities of AL ePNs in an odor-dependent manner and elicits generalized inhibitory effects on ORNs in the AL glomeruli (Dacks, et al., 2009). However, to my knowledge, no study has investigated the modulatory effects of endogenous 5-HT on vPNs, neither the contribution of each receptor types to this modulation. It would be interesting to determine the physiological contribution of serotonergic modulation of vPNs, as well as the contribution of each receptor type to the overall 5-HT-mediated modulation. For instance, one could knock down the 5-HT1A receptor using RNA interference, stimulate the CSD neurons and record from vPNs to observe the change in their activities compared to control flies with full expression of 5-HT1A receptor. Though, it should be noted here that not all vPNs show heterogeneity in terms of their express of the 5-HT receptor types. For instance, only $\sim 21$ vPNs express the receptor 5-HT1A (Sizemore and Dacks, 2014). Therefore, the ability of finding and recordings from the same vPN more than one time could be challenging.

In chapter 3, I demonstrated that reducing the expression of the receptor type 5-HT1A causes an alteration in flies' response to ACV \& BV (Fig. 5F \& 5H). However, when tested for a different innately attractive odor, banana extract, I found no significant difference between the flies that have reduced expression of the receptor type 5-HT1A in their vPNs and control flies with full 5-HT1A vPNs expression (Fig. 5J). This suggests that the 5-HT1A-mediated serotonergic modulation of $\mathrm{vPNs}$ is selective to $\mathrm{ACV}$ and BV (i.e. to odors with specific biological value to the flies). This selectivity could be potentially explained by the heterogeneous nature of the vPNs' and CSD neurons' innervation pattern throughout AL glomeruli, as well as the heterogeneity of vPNs expression of 5-HT receptor types. The ventral cell cluster includes of $\sim 51$ GABAergic vPNs (Lai et., 2008; Strutz et al., 2014), of which $\sim 6$ are labeled by GH146Gal4 line (Jefferis et al., 2001; Wilson and Laurent, 2005; Jefferis et al., 2007) and 45 are labeled by Mz699-Gla4 (Lai et al., 2008; Strutz et al., 2014). The majority of vPNs labeled by 
Mz699 are multiglomerular innervating 5 glomeruli each, and collectively vPNs innervate twothird of AL glomeruli (Strutz et al., 2014). Specifically, vPNs innervate distinct subsets of glomeruli that only respond to attractive odors and are also innervated by ePNs (Strutz et al., 2014). Additionally, the density of Mz699-labeled vPNs dendrites differs between glomeruli (e.g. DM2 and DM5 have dense innervation, while DL4 and DL5 have light innervation) (Strutz et al., 2014). vPNs also show a great diversity in their 5-HT receptor type expression. For instance, vPNs collectively express all 5-HT receptor types, yet, only 21 vPNs express the receptor type 5-HT1A (Sizemore and Dacks, 2016). Furthermore, the innervation of the CSD neurons is heterogeneous across glomeruli. Specifically, the number of CSD synapses differs across glomeruli, such that some glomeruli receive more serotonergic input from CSD neurons than others (Coates et al., in review). The diversity of vPN and CSD neuron innervation of AL glomeruli, as well as the fact that AL glomeruli respond differently to distinctive odors suggest that 5-HT signaling via the 5-HT1A receptor selectively modulates vPNs output in the LH in an odor-dependent manner to change the neural integration of innate attractive odors with different biological values. To further investigate this selectivity, it would be interesting to test odors that elicit responses in different groups of glomeruli which receive different innervation from CSD neurons and vPNs after reducing the expression of 5-HT1 receptors in vPNs.

A second explanation for the absence of 5-HT1A modulation of the banana extract can be suggested based on the data from recent study done by Fisek and Wilson, (2014) in which they discussed a population of LH neurons that are broadly tuned to fruity smelling acetate (such as Isopentyl acetate which is a major component of the banana smell) named Type 1 neurons. Type 1 neurons receive excitatory input from ePNs innervating AL glomeruli that respond to fruitysmelling organic acetate (DM1, DM2, and DM4) and transfer these signals to higher order regions in the brain for the appropriate behavioral responses to be elicited. Unlike vlPrNs, which receive inhibitory input from vPNs, Type $1 \mathrm{LH}$ neurons have been found to receive their major inhibition from a population of GABAergic LH interneurons located adjacent to the $\mathrm{LH}$ and are narrowly tuned to acetate odors (Fisek and Wilson, 2014). This suggests that olfactory information encoding fruity smelling acetate such as IPA and acetoin acetate are potentially selectively processed via a different pathway in the LH that does not rely on inhibition from vPNs. This suggestion could explain why increasing vPN output in the LH by reducing their 5HT1A expression did not show any effects on flies' responses to banana extract in my behavioral 
data. Here, it would be interesting to use the Gal4 line Fisek and Wilson used to identify the LH GABAergic neurons to drive UAS-GAD1-RNAi interference in them to disrupt their GABA synthesis and study the behavioral consequences on flies' attraction to previous odors.

Like aversive odors, vPNs do not respond to pheromone odors (Liang et al., 2013), and thus, serotonergic modulation of vPNs output in the LH is likely not to have any effects on flies' behavioral attraction to pheromone odors. This is suggestion is supported by Zhang and Gaudry, (2016), which showed that endogenous 5-HT release via CSD neuron does not modulate DA1 glomerulus responses to the male pheromone cis-vaccenyl acetate (cVA). However, it would be interesting to further support this hypothesis of the absence of serotonergic modulation of pheromone attraction by doing behavior experiments testing flies responses to cVA after reducing the expression of 5-HT1A in vPNs.

As a conclusion, in this study, I have investigated how a population of neurons that span two processing stages are simultaneously modulated by the same modulatory source in these stages. I showed that vPNs potentially receive a direct axo-dendritic and axo-axonic serotonergic input from CSD neuron in the AL and LH, respectively, which selectively modulate the role of vPNs in flies' responses to odors that have innate importance. Further, I have investigated the degree to which these innate responses are modulated by studying one of the 5 types of 5-HT receptors expressed in vPNs, and via which 5-HT signals to elicit its effects. I have showed that reducing 5-HT1A-mediated inhibition in vPNs increases flies' responses to ACV, but neither to aversive odors nor to fruity-smelling acetate (i.e. banana extract). Collectively, this study provides a better understanding to the mechanistic bases underlying serotonergic modulation of sensory systems and behavior in mammals and insects. 


\section{REFERENCES}

Alekseyenko, O.V., Lee, C., and Kravitz, E.A. (2010). Targeted Manipulation of Serotonergic Neurotransmission Affects the Escalation of Aggression in Adult Male Drosophila melanogaster. PLOS ONE 5, e10806.

Aransay, A., Rodríguez-López, C., García-Amado, M., Clascá, F., and Prensa, L. (2015). Longrange projection neurons of the mouse ventral tegmental area: a single-cell axon tracing analysis. Frontiers in Neuroanatomy 9.

Barrozo, R.B., Gadenne, C., and Anton, S. (2010). Switching attraction to inhibition: matinginduced reversed role of sex pheromone in an insect. Journal of Experimental Biology 213, 2933-2939.

de Belle, J.S., and Heisenberg, M. (1994). Associative odor learning in Drosophila abolished by chemical ablation of mushroom bodies. Science (New York, N.Y.) 263, 692-695.

Blenau, W., and Thamm, M. (2011). Distribution of serotonin (5-HT) and its receptors in the insect brain with focus on the mushroom bodies: lessons from Drosophila melanogaster and Apis mellifera. Arthropod Structure \& Development 40, 381-394.

Brower, M.C., and Price, B.H. (2001). Neuropsychiatry of frontal lobe dysfunction in violent and criminal behaviour: a critical review. Journal of Neurology, Neurosurgery \& Psychiatry 71, 720 726.

Brunert, D., Tsuno, Y., Rothermel, M., Shipley, M.T., and Wachowiak, M. (2016). Cell-TypeSpecific Modulation of Sensory Responses in Olfactory Bulb Circuits by Serotonergic Projections from the Raphe Nuclei. The Journal of Neuroscience: The Official Journal of the Society for Neuroscience 36, 6820-6835.

Byrne, J.H., and Kandel, E.R. (1996). Presynaptic facilitation revisited: state and time dependence. The Journal of Neuroscience: The Official Journal of the Society for Neuroscience $16,425-435$.

Bzdok, D., Laird, A.R., Zilles, K., Fox, P.T., and Eickhoff, S.B. (2013). An investigation of the structural, connectional, and functional subspecialization in the human amygdala. Human Brain Mapping 34, 3247-3266.

Center for History and New Media Zotero Quick Start Guide.

Dacks, A.M., Christensen, T.A., and Hildebrand, J.G. (2006). Phylogeny of a serotoninimmunoreactive neuron in the primary olfactory center of the insect brain. The Journal of Comparative Neurology 498, 727-746.

Dacks, A.M., Christensen, T.A., and Hildebrand, J.G. (2008). Modulation of olfactory information processing in the antennal lobe of Manduca sexta by serotonin. Journal of Neurophysiology 99, 2077-2085.

Dacks, A.M., Green, D.S., Root, C.M., Nighorn, A.J., and Wang, J.W . (2009). Serotonin modulates olfactory processing in the antennal lobe of Drosophila. Journal of Neurogenetics 23, 366-377. 
Davis, R.L. (2005). Olfactory memory formation in Drosophila: from molecular to systems neuroscience. Annual Review of Neuroscience 28, 275-302.

De Belle, J.S., Heisenberg, M., and others (1994). Associative odor learning in Drosophila abolished by chemical ablation of mushroom bodies. Science-AAAS-Weekly Paper EditionIncluding Guide to Scientific Information 263, 692-694.

Farhan, A., Gulati, J., Große-Wilde, E., Vogel, H., Hansson, B.S., and Knaden, M. (2013). The CCHamide 1 receptor modulates sensory perception and olfactory behavior in starved Drosophila. Scientific Reports 3, 2765.

Fişek, M., and Wilson, R.I. (2014). Stereotyped connectivity and computations in higher-order olfactory neurons. Nature Neuroscience 17, 280-288.

Galizia, C.G., and Sachse, S. (2010). Odor Coding in Insects. In The Neurobiology of Olfaction, A. Menini, ed. (Boca Raton (FL): CRC Press/Taylor \& Francis), p.

Gatellier, L., Nagao, T., and Kanzaki, R. (2004). Serotonin modifies the sensitivity of the male silkmoth to pheromone. The Journal of Experimental Biology 207, 2487-2496.

Gnerer, J.P., Venken, K.J.T., and Dierick, H.A. (2015). Gene-specific cell labeling using MiMIC transposons. Nucleic Acids Research 43, e56.

Heimbeck, G., Bugnon, V., Gendre, N., Keller, A., and Stocker, R.F. (2001). A central neural circuit for experience-independent olfactory and courtship behavior in Drosophila melanogaster. Proceedings of the National Academy of Sciences 98, 15336-15341.

Heisenberg, M. (2003). Mushroom body memoir: from maps to models. Nature Reviews Neuroscience 4, 266-275.

Huang, Z., Thiebaud, N., and Fadool, D.A. (2017). Differential serotonergic modulation across the main and accessory olfactory bulbs. The Journal of Physiology.

Hurley, L.M., Devilbiss, D.M., and Waterhouse, B.D. (2004). A matter of focus: monoaminergic modulation of stimulus coding in mammalian sensory networks. Current Opinion in Neurobiology 14, 488-495.

Ito, K., Sass, H., Urban, J., Hofbauer, A., and Schneuwly, S. (1997). GAL4-responsive UAS-tau as a tool for studying the anatomy and development of the Drosophila central nervous system. Cell and Tissue Research 290, 1-10.

Jacobs, B.L., and Azmitia, E.C. (1992). Structure and function of the brain serotonin system. Physiological Reviews 72, 165-229.

Jefferis, G.S.X.E., Marin, E.C., Stocker, R.F., and Luo, L. (2001). Target neuron prespecification in the olfactory map of Drosophila. Nature 414, 204-208.

Jefferis, G.S.X.E., Potter, C.J., Chan, A.M., Marin, E.C., Rohlfing, T., Maurer, C.R., and Luo, L. (2007). Comprehensive Maps of Drosophila Higher Olfactory Centers: Spatially Segregated Fruit and Pheromone Representation. Cell 128, 1187-1203.

Katz, P.S., and Frost, W.N. (1995). Intrinsic neuromodulation in the Tritonia swim CPG: the serotonergic dorsal swim interneurons act presynaptically to enhance transmitter release from interneuron C2. Journal of Neuroscience 15, 6035-6045. 
Katz, P. S. (1999). What are we talking about? In Beyond Neurotransmission: Neuromodualtion and Its Importance for Information processing. New York: Oxford University Press.

Kent, K.S., Hoskins, S.G., and Hildebrand, J.G. (1987). A novel serotonin-immunoreactive neuron in the antennal lobe of the sphinx moth Manduca sexta persists throughout postembryonic life. Journal of Neurobiology 18, 451-465.

Klein, M., Camardo, J., and Kandel, E.R. (1982). Serotonin modulates a specific potassium current in the sensory neurons that show presynaptic facilitation in Aplysia. Proceedings of the National Academy of Sciences of the United States of America 79, 5713-5717.

Kloppenburg, P., and Mercer, A.R. (2008). Serotonin modulation of moth central olfactory neurons. Annual Review of Entomology 53, 179-190.

Kloppenburg, P., Ferns, D., and Mercer, A.R. (1999). Serotonin enhances central olfactory neuron responses to female sex pheromone in the male sphinx moth manduca sexta. The Journal of Neuroscience: The Official Journal of the Society for Neuroscience 19, 8172-8181.

Knaden, M., Strutz, A., Ahsan, J., Sachse, S., and Hansson, B.S. (2012). Spatial Representation of Odorant Valence in an Insect Brain. Cell Reports 1, 392-399.

Knight, D., Xie, W., and Boulianne, G.L. (2011). Neurexins and Neuroligins: Recent Insights from Invertebrates. Molecular Neurobiology 44, 426-440.

Kupfermann, I. (1979). Modulatory actions of neurotransmitters. Annual Review of Neuroscience 2, 447-465.

Kwon, Y., Kim, S.H., Ronderos, D.S., Lee, Y., Akitake, B., Woodward, O.M., Guggino, W.B., Smith, D.P., and Montell, C. (2010). Drosophila TRPA1 Channel Is Required to Avoid the Naturally Occurring Insect Repellent Citronellal. Current Biology 20, 1672-1678.

Lai, S.-L., Awasaki, T., Ito, K., and Lee, T. (2008). Clonal analysis of Drosophila antennal lobe neurons: diverse neuronal architectures in the lateral neuroblast lineage. Development (Cambridge, England) 135, 2883-2893.

Laissue, P.P., and Vosshall, L.B. (2008a). The Olfactory Sensory Map in Drosophila. In Brain Development in Drosophila Melanogaster, G.M. Technau, ed. (Springer New York), pp. 102114.

Laissue, P.P., and Vosshall, L.B. (2008b). The olfactory sensory map in Drosophila. Advances in Experimental Medicine and Biology 628, 102-114.

Liang, L., Li, Y., Potter, C.J., Yizhar, O., Deisseroth, K., Tsien, R.W., and Luo, L. (2013). GABAergic Projection Neurons Route Selective Olfactory Inputs to Specific Higher-Order Neurons. Neuron 79, 917-931.

Linn, C.E., and Roelofs, W.L. (1986). Modulatory effects of octopamine and serotonin on male sensitivity and periodicity of response to sex pheromone in the cabbage looper moth, Trichoplusia ni. Archives of Insect Biochemistry and Physiology 3, 161-171.

Linster, C., and Cleland, T.A. (2016). Neuromodulation of olfactory transformations. Current Opinion in Neurobiology 40, 170-177.

Lottem, E., Lörincz, M.L., and Mainen, Z.F. (2016). Optogenetic Activation of Dorsal Raphe Serotonin Neurons Rapidly Inhibits Spontaneous But Not Odor-Evoked Activity in Olfactory 
Cortex. The Journal of Neuroscience: The Official Journal of the Society for Neuroscience 36, $7-18$.

McLean, J.H., and Shipley, M.T. (1987). Serotonergic afferents to the rat olfactory bulb: I. Origins and laminar specificity of serotonergic inputs in the adult rat. The Journal of Neuroscience: The Official Journal of the Society for Neuroscience 7, 3016-3028.

Mercer, A.R., Hayashi, J.H., and Hildebrand, J.G. (1995). Modulatory effects of 5hydroxytryptamine on voltage-activated currents in cultured antennal lobe neurones of the sphinx moth Manduca sexta. The Journal of Experimental Biology 198, 613-627.

Ng, M., Roorda, R.D., Lima, S.Q., Zemelman, B.V., Morcillo, P., and Miesenböck, G. (2002). Transmission of Olfactory Information between Three Populations of Neurons in the Antennal Lobe of the Fly. Neuron 36, 463-474.

Nichols, D.E., and Nichols, C.D. (2008). Serotonin receptors. Chemical Reviews 108, 16141641 .

Okada, R., Awasaki, T., and Ito, K. (2009). Gamma-aminobutyric acid (GABA)-mediated neural connections in the Drosophila antennal lobe. The Journal of Comparative Neurology 514, 74-91.

Parnas, M., Lin, A.C., Huetteroth, W., and Miesenböck, G. (2013). Odor Discrimination in Drosophila: From Neural Population Codes to Behavior. Neuron 79, 932-944.

Petzold, G.C., Hagiwara, A., and Murthy, V.N. (2009). Serotonergic modulation of odor input to the mammalian olfactory bulb. Nature Neuroscience 12, 784-791.

Root, C.M., Masuyama, K., Green, D.S., Enell, L.E., Nässel, D.R., Lee, C.-H., and Wang, J.W. (2008). A presynaptic gain control mechanism fine-tunes olfactory behavior. Neuron 59, 311321.

Saudou, F., Boschert, U., Amlaiky, N., Plassat, J.L., and Hen, R. (1992). A family of Drosophila serotonin receptors with distinct intracellular signalling properties and expression patterns. The EMBO Journal 11,7-17.

Saveer, A.M., Kromann, S.H., Birgersson, G., Bengtsson, M., Lindblom, T., Balkenius, A., Hansson, B.S., Witzgall, P., Becher, P.G., and Ignell, R. (2012). Floral to green: mating switches moth olfactory coding and preference. Proceedings. Biological Sciences 279, 2314-2322.

Semmelhack, J.L., and Wang, J.W . (2009). Select Drosophila glomeruli mediate innate olfactory attraction and aversion. Nature 459, 218-223.

Shimada-Niwa, Y., and Niwa, R. (2014). Serotonergic neurons respond to nutrients and regulate the timing of steroid hormone biosynthesis in Drosophila. Nature Communications 5, 5778.

Silbering, A.F., Okada, R., Ito, K., and Galizia, C.G. (2008). Olfactory information processing in the Drosophila antennal lobe: anything goes? The Journal of Neuroscience: The Official Journal of the Society for Neuroscience 28, 13075-13087.

Sizemore, T.R., and Dacks, A.M. (2016). Serotonergic Modulation Differentially Targets Distinct Network Elements within the Antennal Lobe of Drosophila melanogaster. Scientific Reports 6,37119. 
Stensmyr, M.C., Dweck, H.K.M., Farhan, A., Ibba, I., Strutz, A., Mukunda, L., Linz, J., Grabe, V., Steck, K., Lavista-Llanos, S., et al. (2012). A Conserved Dedicated Olfactory Circuit for Detecting Harmful Microbes in Drosophila. Cell 151, 1345-1357.

Stocker, R.F., Heimbeck, G., Gendre, N., and de Belle, J.S. (1997a). Neuroblast ablation in Drosophila P[GAL4] lines reveals origins of olfactory interneurons. Journal of Neurobiology 32, 443-456.

Stocker, R.F., Heimbeck, G., Gendre, N., and de Belle, J.S. (1997b). Neuroblast ablation in Drosophila P[GAL4] lines reveals origins of olfactory interneurons. Journal of Neurobiology 32, 443-456.

Strawn, J.R., and Geracioti, T.D. (2008). Noradrenergic dysfunction and the psychopharmacology of posttraumatic stress disorder. Depression and Anxiety 25, 260-271.

Strutz, A., Soelter, J., Baschwitz, A., Farhan, A., Grabe, V., Rybak, J., Knaden, M., Schmuker, M., Hansson, B.S., and Sachse, S. (2014). Decoding odor quality and intensity in the Drosophila brain. eLife 3, e04147.

Tanaka, N.K., Endo, K., and Ito, K. (2012). Organization of antennal lobe-associated neurons in adult Drosophila melanogaster brain. The Journal of Comparative Neurology 520, 4067-4130.

Vosshall, L.B., Wong, A.M., and Axel, R. (2000a). An Olfactory Sensory Map in the Fly Brain. Cell 102, 147-159.

Vosshall, L.B., Wong, A.M., and Axel, R. (2000b). An Olfactory Sensory Map in the Fly Brain. Cell 102, 147-159.

Wang, J., Zugates, C.T., Liang, I.H., Lee, C.-H.J., and Lee, T. (2002). Drosophila Dscam is required for divergent segregation of sister branches and suppresses ectopic bifurcation of axons. Neuron 33, 559-571.

Wang, J.W., Wong, A.M., Flores, J., Vosshall, L.B., and Axel, R. (2003). Two-photon calcium imaging reveals an odor-evoked map of activity in the fly brain. Cell 112, 271-282.

Wang, K., Gong, J., Wang, Q., Li, H., Cheng, Q., Liu, Y., Zeng, S., and Wang, Z. (2014). Parallel pathways convey olfactory information with opposite polarities in Drosophila. Proceedings of the National Academy of Sciences 111, 3164-3169.

Weiger, W.A. (1997). Serotonergic modulation of behaviour: a phylogenetic overview. Biological Reviews of the Cambridge Philosophical Society 72, 61-95.

Wilson, R.I., and Laurent, G. (2005). Role of GABAergic inhibition in shaping odor-evoked spatiotemporal patterns in the Drosophila antennal lobe. The Journal of Neuroscience: The Official Journal of the Society for Neuroscience 25, 9069-9079.

Wilson, R.I., Turner, G.C., and Laurent, G. (2004). Transformation of Olfactory Representations in the Drosophila Antennal Lobe. Science 303, 366-370.

Witz, P., Amlaiky, N., Plassat, J.L., Maroteaux, L., Borrelli, E., and Hen, R. (1990). Cloning and characterization of a Drosophila serotonin receptor that activates adenylate cyclase. Proceedings of the National Academy of Sciences of the United States of America 87, 8940-8944.

Zhang, X., and Gaudry, Q. (2016). Functional integration of a serotonergic neuron in the Drosophila antennal lobe. eLife 5. 
Zhang, Y.Q., Rodesch, C.K., and Broadie, K. (2002). Living synaptic vesicle marker: synaptotagmin-GFP. Genesis (New York, N.Y.: 2000) 34, 142-145.

Zhu, J., Park, K.-C., and Baker, T.C. (2003). Identification of odors from overripe mango that attract vinegar flies, Drosophila melanogaster. Journal of Chemical Ecology 29, 899-909.

Raguso RA, Henzei C, Buchmann SL, Nabhan GP. Trumpet flowers of the Sonoran desert: floral biology of Peniocereus cacti and sacred Datura. International Journal of Plant Sciences. 